\title{
Bounds for the energy of a complex unit gain graph*
}

\author{
Aniruddha Samanta $^{\dagger} \quad$ M. Rajesh Kannan ${ }^{\ddagger}$
}

May 19, 2020

\begin{abstract}
A $\mathbb{T}$-gain graph, $\Phi=(G, \varphi)$, is a graph in which the function $\varphi$ assigns a unit complex number to each orientation of an edge, and its inverse is assigned to the opposite orientation. The associated adjacency matrix $A(\Phi)$ is defined canonically. The energy $\mathcal{E}(\Phi)$ of a $\mathbb{T}$-gain graph $\Phi$ is the sum of the absolute values of all eigenvalues of $A(\Phi)$. We study the notion of energy of a vertex of a $\mathbb{T}$-gain graph, and establish bounds for it. For any $\mathbb{T}$-gain graph $\Phi$, we prove that $2 \tau(G)-2 c(G) \leq \mathcal{E}(\Phi) \leq$ $2 \tau(G) \sqrt{\Delta(G)}$, where $\tau(G), c(G)$ and $\Delta(G)$ are the vertex cover number, the number of odd cycles and the largest vertex degree of $G$, respectively. Furthermore, using the properties of vertex energy, we characterize the classes of $\mathbb{T}$-gain graphs for which $\mathcal{E}(\Phi)=2 \tau(G)-2 c(G)$ holds. Also, we characterize the classes of $\mathbb{T}$-gain graphs for which $\mathcal{E}(\Phi)=2 \tau(G) \sqrt{\Delta(G)}$ holds. This characterization solves a general version of an open problem. In addition, we establish bounds for the energy in terms of the spectral radius of the associated adjacency matrix.
\end{abstract}

AMS Subject Classification(2010): 05C50, 05C22, 05C35.

\section{Introduction}

In a simple undirected graph $G$ with vertex set $V(G)=\left\{v_{1}, \ldots, v_{n}\right\}$ and edge set $E(G)$, if two vertices $v_{p}$ and $v_{q}$ are adjacent in $G$, then we write $v_{p} \sim v_{q}$, and the edge in between them is denoted by $e_{p, q}$. The number of vertices adjacent with the vertex $v_{p}$, the degree of $v_{p}$, is denoted by $d\left(v_{p}\right)$ (or simply $d_{p}$ ). $\Delta(G)$ denotes the maximum vertex degree of $G$. A directed graph(or digraph) $X$ is an order pair $(V(X), E(X))$, where

*This paper is dedicated to Professor Ravindra Bhalchandra Bapat on the occasion of his 65th birthday with much admiration.

${ }^{\dagger}$ Department of Mathematics, Indian Institute of Technology Kharagpur, Kharagpur 721302, India. Email: aniruddha.sam@gmail.com

${ }^{\ddagger}$ Department of Mathematics, Indian Institute of Technology Kharagpur, Kharagpur 721302, India. Email: rajeshkannan@maths.iitkgp.ac.in, rajeshkannan1.m@gmail.com 
$V(X)=\left\{v_{1}, v_{2}, \ldots, v_{n}\right\}$ is the vertex set and $E(X)$ is the directed edge set. A directed edge from the vertex $v_{p}$ to the vertex $v_{q}$ is denoted by $\overrightarrow{e_{p, q}}$. If $\overrightarrow{e_{p, q}} \in E(X)$ and $\overrightarrow{e_{p, q}} \in E(X)$, then the pair $\left\{v_{p}, v_{q}\right\}$ is called a digon of $X$. The Hermitian adjacency matrix [4, 9] of a digraph $X$ is denoted by $H(X)$ and is defined as follows:

$$
(p, q) \text { th entry of } H(X)=h_{p, q}= \begin{cases}1 & \text { if both } \overrightarrow{e_{p, q}} \text { and } \overrightarrow{e_{q, p}} \in E(X), \\ i & \text { if } \overrightarrow{e_{p, q}} \in E(X) \text { and } \overrightarrow{e_{q, p}} \notin E(X), \\ -i & \text { if } \overrightarrow{e_{p, q}} \notin E(X) \text { and } \overrightarrow{e_{q, p}} \in E(X), \\ 0 & \text { otherwise. }\end{cases}
$$

The Hermitian adjacency matrix can be thought of as the adjacency matrix of a $\mathbb{T}$-gain graph with the gains are from the set $\{1, \pm i\}$. A digraph is said to be an oriented graph if it has no digons. A graph contains both directed and undirected edges is called a mixed graph and it is denoted by $D_{G}$, where $G$ is the underlying simple graph. When we consider Hermitian adjacency matrix, $H\left(D_{G}\right)$ of a mixed graph $D_{G}$, the undirected edges are treated as digons.

From a simple graph $G$, by orienting each undirected edge $e_{p, q} \in E(G)$ in two opposite directions, namely $\overrightarrow{e_{p, q}}$ and $\overrightarrow{e_{q, p}}$, we get a digraph. Let $\overrightarrow{E(G)}=\left\{\overrightarrow{e_{p, q}}, \overrightarrow{e_{q, p}}: e_{p, q} \in E(G)\right\}$ and $\mathbb{T}=\{z \in \mathbb{C}:|z|=1\}$. A complex unit gain graph (simply, $\mathbb{T}$-gain graph) on a simple graph $G$ is a pair $(G, \varphi)$, where $\varphi: \overrightarrow{E(G)} \rightarrow \mathbb{T}$ is a mapping such that $\varphi\left(\overrightarrow{e_{p, q}}\right)=\varphi\left(\overrightarrow{e_{q, p}}\right)^{-1}$. A $\mathbb{T}$-gain graph $(G, \varphi)$ is denoted by $\Phi$. For more details about the $\mathbb{T}$-gain graphs, we refer to [10, 11, 12, 13, 18].

The adjacency matrix of $\Phi$ is the Hermitian matrix $A(\Phi)=\left(a_{p, q}\right)_{n \times n}$ defined as follows:

$$
a_{p, q}= \begin{cases}\varphi\left(\overrightarrow{e_{p, q}}\right) & \text { if } v_{p} \sim v_{q} \\ 0 & \text { otherwise }\end{cases}
$$

Let $\left\{\lambda_{1}, \ldots, \lambda_{n}\right\}$ be the spectrum of $A(\Phi)$ (or the spectrum of $\Phi$ ), and is denoted by $\operatorname{spec}(\Phi)$. The energy of $\Phi$, denoted by $\mathcal{E}(\Phi)$, is defined by $\sum_{j=1}^{n}\left|\lambda_{j}\right|$.

For a vertex $v_{j}$ of $G$, the energy of the vertex $v_{j}$, denoted by $\mathcal{E}_{G}\left(v_{j}\right)$, is defined by $\mathcal{E}_{G}\left(v_{j}\right)=|A(G)|_{j j}$, where $|A(G)|=\left(A(G) A(G)^{*}\right)^{\frac{1}{2}}$ and $|A(G)|_{j j}$ is the $(j, j)$-th entry of $|A(G)|$. Then $\mathcal{E}(G)=\sum_{j=1}^{n} \mathcal{E}_{G}\left(v_{j}\right)$ [1]. In Section 3 , we establish bounds for $\mathcal{E}_{\Phi}\left(v_{j}\right)$, the vertex energy of a $\mathbb{T}$-gain graph, in terms degree of the vertex $v_{j}$, and characterize the classes of graphs for which the bounds are sharp. As a consequence of these bounds, we provide a couple of bounds for the energy of a $\mathbb{T}$-gain graph in terms of the energy of the underlying graph and the number of vertices of the graph. 
A matching in a graph $G$ is a set of edges of $G$ such that no two edges are incident with the same vertex. The cardinality of a matching with the maximum number of edges is the matching number of $G$, and is denoted by $\mu(G)$. A matching that saturates all the vertices of $G$ is known as a perfect matching of $G$. A vertex cover $U$ of a graph $G$ is a subset of $V(G)$ such that every edge of $G$ is incident with at least one vertex of $U$. The cardinality of a vertex cover with the minimum number of vertices is the vertex cover number of $G$, and is denoted by $\tau(G)$. For any $\mathbb{T}$-gain graph $\Phi=(G, \varphi)$, the matching number, and the vertex cover number of $\Phi$ are the matching number and the vertex cover number of the underlying graph $G$, respectively.

In [15], the authors derived a lower bound for the energy of an undirected graph in terms of the vertex cover number and the number of odd cycles.

Theorem 1.1 ([15, Theorem 4.2]). If $G$ is a graph with the vertex cover number $\tau(G)$ and the number of odd cycle $c(G)$, then $\mathcal{E}(G) \geq 2 \tau(G)-2 c(G)$. Equality occurs if and only if each component of $G$ is a complete bipartite graph with perfect matching together with some isolated vertices.

In [16], the authors extended Theorem 1.1 for Hermitian adjacency matrices of mixed graphs.

Theorem 1.2 ([16, Theorem 4.5]). Let $D_{G}$ be a mixed graph with vertex cover number $\tau(G)$ and number of odd cycles $c(G)$. Then $\mathcal{E}_{H}\left(D_{G}\right) \geq 2 \tau(G)-2 c(G)$. Equality occurs if and only if $D_{G}$ is switching equivalent to its underlying graph $G$, where each component of $G$ is either a complete bipartite graph with equal partition size or isolated vertices.

Further extensions of Theorem 1.1 are discussed in [14, 17].

In Section 4, we obtain lower bounds for $\mathcal{E}(\Phi)$ in terms of the gains of fundamental cycles [Theorem 4.1 and Theorem 4.4]. We show that a connected $\mathbb{T}$-gain bipartite graph has exactly one positive eigenvalue if and only if it is the balanced complete bipartite graph [Theorem 4.2 . We establish a bound for the energy of a $\mathbb{T}$-gain graph in terms of the spectral radius of $\Phi$, and characterize the sharpness of the inequality [Theorem 4.3]. Further, we establish lower bounds for $\mathcal{E}(\Phi)$ in terms of the vertex cover number, the number of odd cycles, and the matching number [Theorem 4.7 and Theorem 4.8. After completion of this work, we learned that Theorem 4.7 has been proved in [8] independently. However, our proof uses the properties of vertex energy of $\mathbb{T}$-gain graphs, and hence the proof is different from the proof given in [8]. 
In [15], the authors established an upper bound of the energy of an undirected graph in terms of the vertex cover number and the largest vertex degree.

Theorem 1.3. [15, Theorem 3.1] If $G$ is an undirected graph with vertex cover number $\tau(G)$ and maximum vertex degree $\Delta(G)$, then $\mathcal{E}(G) \leq 2 \tau(G) \sqrt{\Delta(G)}$. Equality occurs if and only if $G$ is the disjoint union of $\tau(G)$ copies of $K_{1, \Delta(G)}$ together with some isolated vertices.

In [16], the authors extended this inequality for a mixed graph and proposed the equality part as an open problem.

Theorem 1.4 ([16, Theorem 4.9]). Let $D_{G}$ be a mixed graph with vertex cover number $\tau(G)$ and largest vertex degree $\Delta(G)$. Then

$$
\mathcal{E}_{H}\left(D_{G}\right) \leq 2 \tau(G) \sqrt{\Delta(G)}
$$

In Section 5 , we extend Theorem 1.4 for the $\mathbb{T}$-gain graphs [Theorem 5.1 .

Problem 1.1 ([16, Problem 4.1]). Characterize all mixed graphs which make the equality in (1) hold.

We solve this problem for the $\mathbb{T}$-gain graphs [Theorem 5.2]. The Hermitian adjacency matrices of mixed graphs are particular cases of the adjacency matrices of the $\mathbb{T}$-gain graphs. Also, in a recent manuscript [8], the author mentioned the difficulties in extending Theorem 1.4 , and characterizing the graphs for which equality hold for the $\mathbb{T}$-gain graphs.

This article is organized as follows: In Section 2, we collect needed known definitions and results. In Section 3, we extend the notion of vertex energy for $\mathbb{T}$-gain graphs, and establish some of the properties. In Section 4, we establish various lower bounds for the energy of $\mathbb{T}$-gain graphs, and Section 5 is devoted to upper bounds for the energy of $\mathbb{T}$-gain graphs.

\section{Definitions, notation and preliminary results}

In this section we recall some of the needed graph theory and linear algebra terminologies and some of the basic results. A subgraph $H$ of a graph $G$ is an induced subgraph if two vertices of $H$ are adjacent in $G$, then they are adjacent in $H$. For an induced subgraph $H$ of $G$ the complement of $H$ in $G$, denoted by $G-H$, defined as the induced subgraph of $G$ with vertex set $V(G) \backslash V(H)$. The subgraphs $H$ and $G-H$ are called complementary induced subgraphs in $G$. If $E$ is any edge set of $G$, then $G-E$ denotes the spanning subgraph of $G$ with edge set $E(G) \backslash E$ and vertex set $V(G)$. A cut of a graph $G$ is a partition of the vertex set $V(G)$ into 
two sets $U$ and $W$. A cut set of $G$ is a set of edges $\left\{e_{p, q} \in E(G): v_{p} \in U, v_{q} \in W\right\}$, where $U$ and $V$ partition the vertex set $V(G)$. Suppose $E$ is a cut set, then there are two induced subgraphs $H$ and $G-H$ complement to each other such that each edge of $E$ is incident to a vertex of $H$ and to another vertex of $G-H$ [3]. Then we denote $H \oplus(G-H)=G-E$.

Let $e_{p, q} \in E(G)$. To avoid confusion, we denote $G-\left[e_{p, q}\right]$ as an induced subgraph of $G$ whose vertex set is $V(G) \backslash\left\{v_{p}, v_{q}\right\}$. If $K$ is a spanning subgraph of $G$, then for any edge $e \in E(G) \backslash E(K), K+e$ denotes a spanning subgraph of $G$ with the edge set $E(K) \cup\{e\}$. If $G$ is a connected graph and $T$ is a spanning tree of $G$, then any edge $e \in E(G) \backslash E(T)$ induces a unique cycle in $T+e$. This is called a fundamental cycle in $G$ with respect to $T$.

The adjacency matrix of a simple graph $G$, denoted by $A(G)$, is the symmetric $n \times n$ matrix whose $(p, q)$ th entry is defined by $a_{p, q}=1$ if $v_{p} \sim v_{q}$, and $a_{p, q}=0$ otherwise. The energy of the graph $G$, denoted by $\mathcal{E}(G)$, is the sum of the absolute values of the eigenvalues of $A(G)$.

Lemma 2.1 ([3, Theorem 3.6]). Let $L$ and $M$ be two complementary induced subgraph of a graph $G$ and $E$ be the cut set in between them. If $E$ is not empty and all edges in $E$ are incident to one and only one vertex in $M$, then $\mathcal{E}(G-E)<\mathcal{E}(G)$.

Let $\Phi=(G, \varphi)$ be any $\mathbb{T}$-gain graph, and $H$ be a subgraph of $G$. We call $(H, \xi)$ a subgraph of $\Phi$ if the function $\xi$ is the restriction of $\varphi$ on $\overrightarrow{E(H)}$, and is denoted by $(H, \varphi)$ (instead of $(H, \xi)$ ). If $H$ is an induced subgraph of $G$ and $E$ is any edge set of $G$, then similar to undirected graphs we can define $\Phi-H$ and $\Phi-E$.

The adjacency matrix of $\Phi=(G, \varphi)$, denoted by $A(\Phi)$, is defined as the Hermitian matrix whose $(p, q)$-th element is $\varphi\left(\overrightarrow{e_{p, q}}\right)$ if $v_{p} \sim v_{q}$ and, zero otherwise. The spectrum of $\Phi$, denoted by $\operatorname{spec}(\Phi)$, is the spectrum of $A(\Phi)$. The spectral radius of $\Phi$ is denoted by $\rho(\Phi)$. The energy of $\Phi$, denoted by $\mathcal{E}(\Phi)$, is defined as $\mathcal{E}(\Phi)=\sum_{j=1}^{n}\left|\lambda_{j}\right|$, where $\lambda_{j}$ are the eigenvalues of $\Phi$. Two $\mathbb{T}$-gain graphs $\Phi=(G, \varphi)$ and $\Phi^{\prime}=\left(G, \varphi^{\prime}\right)$ are switching equivalent if there exists a unitary diagonal matrix $U$ such that $A\left(\Phi^{\prime}\right)=U A(\Phi) U^{*}$. If $\Phi$ and $\Phi^{\prime}$ are switching equivalent, then it is denoted by $\Phi \sim \Phi^{\prime}$.

A directed cycle is called an oriented cycle if all of its edges are directed such that each edge is traversed in the same direction. An undirected cycle of $k$ vertices $C \equiv v_{1}-v_{2}-$ $\cdots-v_{k}-v_{1}$ has two oriented cycles. If one of the orientation, say $v_{1} \rightarrow v_{2} \rightarrow \ldots v_{k} \rightarrow v_{1}$, is denoted by $\vec{C}$, then opposite oriented cycle is denoted by $\vec{C}^{*}$. The gain of an oriented cycle $\vec{C}$ is defined as $\varphi(\vec{C})=\varphi\left(\overrightarrow{e_{1,2}}\right) \varphi\left(\overrightarrow{e_{2,3}}\right) \cdots \varphi\left(\overrightarrow{e_{k, 1}}\right)$. Therefore, $\varphi\left(\vec{C}^{*}\right)=\{\varphi(\vec{C})\}^{-1}$. For any complex number $\lambda, \operatorname{Re}(\lambda)$ denotes the real part of $\lambda$. If $\varphi(\vec{C})=\varphi\left(\vec{C}^{*}\right)=1$, then we simply 
write $\varphi(C)=1$. Similarly, for any cycle $C, \operatorname{Re}(\varphi(\vec{C}))=\operatorname{Re}\left(\varphi\left(\vec{C}^{*}\right)\right)$. Thus, we simple write $\operatorname{Re}(\varphi(C))$.

A $\mathbb{T}$-gain graph $\Phi=(G, \varphi)$ is called balanced if $\varphi(\vec{C})=1$, for any cycle $C$ in $G$. If $\Phi$ is balanced, then $\Phi \sim(G, 1)$. Some of the properties of $\mathbb{T}$-gain graphs are collected in the next couple of results.

Theorem 2.1 ( [10, Lemma 4.1, Theorem 4.4]). Let $\Phi=(G, \varphi)$ be any $\mathbb{T}$-gain graph on a connected graph $G$. Then $\rho(\Phi) \leq \rho(G)$. Equality occur if and only if either $\Phi$ or $-\Phi$ is balanced.

Theorem 2.2 ([10, Theorem 4.5] ). Let $G$ be a connected graph. Then we have the following:

1. If $G$ is bipartite, then whenever $\Phi$ is balanced implies $-\Phi$ is balanced.

2. If $\Phi$ is balanced implies $-\Phi$ is balanced for some gain, then $G$ is bipartite.

Lemma $2.2\left(\left[13\right.\right.$, Corollary 3.2]). Let $\Phi_{1}=\left(G, \varphi_{1}\right)$ and $\Phi_{2}=\left(G, \varphi_{2}\right)$ be two $\mathbb{T}$-gain graphs on a connected graph $G$ with $n$ vertices and $m$ edges. Let $\left\{C_{1}, C_{2}, \ldots, C_{m-n+1}\right\}$ be the fundamental cycles of $G$ with respect to a normal spanning tree of $G$. Then $\Phi_{1} \sim \Phi_{2}$ if and only if $\varphi_{1}\left(\overrightarrow{C_{j}}\right)=\varphi_{2}\left(\overrightarrow{C_{j}}\right)$, for all $j=1,2, \ldots,(m-n+1)$.

Let $C_{n}$ denote the cycle on $n$ vertices.

Theorem 2.3 ([11, Theorem 6.1]). Let $\Phi=\left(C_{n}, \varphi\right)$ be a $\mathbb{T}$-gain graph with $\varphi\left(\overrightarrow{C_{n}}\right)=e^{i \theta}$. Then

$$
\operatorname{spec}(\Phi)=\left\{2 \cos \left(\frac{\theta+2 \pi j}{n}\right): j=0,1, \ldots,(n-1)\right\} .
$$

Lemma 2.3 ( [6, Theorem 1.13]). Let $\Phi=(G, \varphi)$ be any connected $\mathbb{T}$-gain graph. Then

$$
2 \max _{V_{0}} \mu\left(G-V_{0}\right) \leq r(G, \varphi) \leq 2 \mu(G)+b(G),
$$

where $V_{0}$ is any proper subset of $V(G)$ such that $G-V_{0}$ is acyclic and $b(G)$ is the minimum integer $|U|$ such that $G-U$ is bipartite, $U \subset V(G)$.

In [1], the authors studied the notion of vertex energy of a graph.

Definition 2.1 ([1, Definition 2.1]). Let $G$ be a graph with vertex set $V(G)=\left\{v_{1}, v_{2}, \ldots, v_{n}\right\}$. Then the energy of a vertex $v_{j}$, denoted by $\mathcal{E}_{G}\left(v_{j}\right)$, is defined as $\mathcal{E}_{G}\left(v_{j}\right)=|A(G)|_{j j}$, where $|A(G)|=\left(A(G) A(G)^{*}\right)^{\frac{1}{2}}$. 
Next, we recall a few results related to the vertex energy.

Lemma 2.4 ([1, Lemma 2.2]). Let $G$ be an undirected graph with vertex set $V(G)=$ $\left\{v_{1}, v_{2}, \ldots, v_{n}\right\}$. Then

$$
\mathcal{E}_{G}\left(v_{i}\right)=\sum_{j=1}^{n} Q_{i j}\left|\lambda_{j}\right|, \text { for } i=1,2, \ldots n .
$$

where $Q_{i j}=q_{i j}^{2}$ and $Q=\left(q_{i j}\right)$ is the orthogonal matrix whose columns are the eigenvectors of $G$ and $\lambda_{j}$ is the $j$-th eigenvalue of $G$.

Lemma 2.5 ([1, Theorem 3.3]). If $G$ is a connected graph on $n$ vertices with at least one edge, then

$$
\mathcal{E}_{G}\left(v_{j}\right) \geq \frac{d_{j}}{\Delta(G)}, \quad \text { for all } v_{j} \in V(G) .
$$

Equality occurs if and only if $G$ is a complete bipartite graph with equal partition size.

Let $G$ and $G_{1}$ be two simple graphs. Let $D_{G}$ be a mixed graph on $G$. The mixed Kronecker product, denoted by $D_{G} \otimes G_{1}$, is the Kronecker product of the Hermitian adjacency matrix of $G$ and the adjacency matrix of the simple graph $G_{1}$ [16].

Lemma 2.6 ([16, Lemma 2.7]). Let $\left\{\lambda_{1}, \lambda_{2}, \ldots, \lambda_{s}\right\}$ be the spectrum of $G_{1}$, and $\left\{\gamma_{1}, \gamma_{2}, \ldots, \gamma_{t}\right\}$ be the spectrum of $D_{G}$ (with respect to the Hermitian adjacency matrix), then the spectrum of a mixed Kronecker product $D_{G} \otimes G_{1}$ is $\left\{\lambda_{i} \gamma_{j}: 1 \leq i \leq s, 1 \leq j \leq t\right\}$

The Hermitian energy of a mixed graph $D_{G}$ is the sum of the absolute values of the eigenvalues of $H\left(D_{G}\right)$, and is denoted by $\mathcal{E}_{H}\left(D_{G}\right)$.

Let us collect a few results on energy in terms of matching number.

Lemma 2.7 ([15, Lemma 4.1]). For any bipartite graph $G, \mathcal{E}(G) \geq 2 \mu(G)$. Equality occur if and only if each component of $G$ is complete bipartite graph with perfect matching together with some isolated vertices.

Theorem 2.4 ([17, Theorem 1.1]). Let $G$ be a graph with matching number $\mu(G)$. Then $\mathcal{E}(G) \geq 2 \mu(G)$. If all cycles (if any) of $G$ are pairwise vertex disjoint, then equality holds if and only if each component of $G$ is either an edge or 4-cycle or an isolated vertices.

Theorem 2.5 ([16, Theorem1.1, Theorem 1.2]). Let $D_{G}$ be a mixed graph with matching number $\mu(G)$, then $\mathcal{E}_{H}\left(D_{G}\right) \geq 2 \mu(G)$. Equality occur if and only if $D_{G}$ is switching equivalent to its underlying graph $G$, where each component of $G$ is either a complete bipartite graph with equal partition size or isolated vertices. 
Lemma 2.8 ([16, Lemma 3.8]). Let $D_{G}$ be a mixed graph on a connected non bipartite graph G. Then $\mathcal{E}_{H}\left(D_{G}\right)>2 \mu(G)$.

Lemma 2.9 ([16, Lemma 3.6]). Let $D_{G}$ be a mixed graph without isolated vertices. If $\mathcal{E}_{H}\left(D_{G}\right)=2 \mu(G)$, then $G$ has a perfect matching.

A graph $G$ is bipartite graph if its vertex set $V(G)$ can be partitioned into two sets, $X$ and $Y$ such that every edge of $G$ joins a vertex of $X$ with a vertex of $Y$. If every vertex in $X$ is adjacent to every vertex in $Y$, then the graph $G$ is called a complete bipartite graph. If $G$ is a complete bipartite graph with $|X|=p$ and $|Y|=q$, then $G$ is denoted by $K_{p, q}$. For instance, $K_{p, p}$ is a complete bipartite graph with a perfect matching. A graph $G$ is called an $r$-regular graph (or regular graph) if every vertex of $G$ has the same degree $r$. A graph $G$ is called a semiregular bipartite graph with parameter $\left(n_{a}, n_{b}, r_{a}, r_{b}\right)$ if $G$ is a bipartite graph with $|X|=n_{a}$ and $|Y|=n_{b}$ such that all the vertices of $X$ have the same degree $r_{a}$, and the vertices of $Y$ have the same degree $r_{b}$.

Theorem 2.6 ([5, Theorem 3]). If $G$ is a d-regular graph of $n$ vertices, then $\mathcal{E}(G) \geq n$. Equality holds if and only if each component is isomorphic to $K_{d, d}$.

Let $G$ be a semiregular bipartite graph with partition size $n_{a}$ and $n_{b}$, and the vertex degree of each vertex of first and second partition is $r_{a}$ and $r_{b}$, respectively. The next result provides a bound of $\mathcal{E}(G)$.

Theorem 2.7 ([5, Theorem 5]). If $G$ is a semiregular graph with the parameter $\left(n_{a}, n_{b}, r_{a}, r_{b}\right)$. Then $\mathcal{E}(G) \geq n_{a} \sqrt{\frac{r_{a}}{r_{b}}}+n_{b} \sqrt{\frac{r_{b}}{r_{a}}}$ and equality occur if and only if every component of $G$ is $K_{r_{a}, r_{b}}$.

For an $n \times n$ complex square matrix $A$, $\operatorname{trace}(A)$ denotes the trace of the matrix $A$. The next result is known as the von Neumann's trace theorem.

Theorem $2.8([7])$. Let $A$ and $B$ be two square complex matrices with singular values $\lambda_{1}(A) \geq \lambda_{2}(A) \geq \cdots \geq \lambda_{n}(A)$ and $\lambda_{1}(B) \geq \lambda_{2}(B) \geq \cdots \geq \lambda_{n}(B)$, respectively. Then

$$
\operatorname{Re}(\operatorname{trace}(A B)) \leq \sum_{j=1}^{n} \lambda_{j}(A) \lambda_{j}(B) .
$$

Theorem 2.9 ([3, Corollary 2.4.]). If $A=\left[\begin{array}{cc}B & X \\ Y & C\end{array}\right]$ is any partition matrix with $A$ and $B$ are the square matrices, then $\mathcal{E}(A) \geq \mathcal{E}(B)$. Equality occurs if and only if $X, Y$ and $C$ are all zero matrices. 
Theorem 2.10 ([3, Theorem 2.2]). Let $A=\left[\begin{array}{ll}A_{11} & A_{12} \\ A_{21} & A_{22}\end{array}\right]$ be a complex block matrix such that both the diagonal blocks are square matrices. Then $\mathcal{E}\left(A_{11}\right)+\mathcal{E}\left(A_{22}\right) \leq \mathcal{E}(A)$, where $\mathcal{E}(A)$ is the sum of the singular values of $A$. Equality occurs if and only if there exist unitary matrices $U$ and $V$ such that $\left[\begin{array}{ll}U A_{11} & U A_{12} \\ V A_{21} & V A_{22}\end{array}\right]$ is positive semidefinite.

Theorem 2.11. [2] Let $C, C_{1}$ and $C_{2}$ be three square complex matrices of order $n$ such that $C=C_{1}+C_{2}$. If $S_{j}(\cdot)$ is the $j$-th singular value of corresponding matrix, then $\sum_{p} S_{p}(C) \leq \sum_{p} S_{p}\left(C_{1}\right)+\sum_{p} S_{p}\left(C_{2}\right)$.

\section{Energy of a vertex of $\mathbb{T}$-gain graphs}

The energy of a vertex in an undirected graph is studied in [1]. In this section, first we extend this notion for the $\mathbb{T}$-gain graphs, and establish some of the properties.

Definition 3.1. The energy of a vertex $v_{i}$ of a $\mathbb{T}$-gain graph $\Phi$ is denoted by $\mathcal{E}_{\Phi}\left(v_{i}\right)$ and is defined by

$$
\mathcal{E}_{\Phi}\left(v_{i}\right)=|A(\Phi)|_{i i}, \text { for } i=1,2 \ldots, n,
$$

where $|A(\Phi)|_{i i}$ is the $(i, i)$-th entry of $\left(A(\Phi) A(\Phi)^{*}\right)^{\frac{1}{2}}$.

It is easy to see that, the energy of a $\mathbb{T}$-gain graph can be expressed as the sum of the energies of vertices of $\Phi$. That is,

$$
\mathcal{E}(\Phi)=\mathcal{E}_{\Phi}\left(v_{1}\right)+\mathcal{E}_{\Phi}\left(v_{2}\right)+\cdots+\mathcal{E}_{\Phi}\left(v_{n}\right)
$$

Energy of a vertex of a $\mathbb{T}$-gain graph can be obtained from the eigenvalues and the eigenvectors of $\Phi$. This is done in the next Lemma, and this result is an extension of Lemma 2.4 for the $\mathbb{T}$-gain graphs.

Lemma 3.1. Let $\Phi$ be a $\mathbb{T}$-gain graph with the vertex set $\left\{v_{1}, v_{2}, \ldots, v_{n}\right\}$. Then

$$
\mathcal{E}_{\Phi}\left(v_{i}\right)=\sum_{j=1}^{n} Q_{i j}\left|\lambda_{j}\right|, \text { for } i=1,2, \ldots n .
$$

where $Q_{i j}=\left|q_{i j}\right|^{2}$ and $Q=\left(q_{i j}\right)$ is the unitary matrix whose columns are the eigenvectors of $\Phi$ and $\lambda_{j}$ is the $j$-th eigenvalue of $\Phi$. 
Proof. Since $A(\Phi)$ is Hermitian, so there exists a unitary matrix $Q=\left(q_{i j}\right)$ such that $A(\Phi)=$ $Q D Q^{*}$, where $D=\operatorname{diag}\left(\lambda_{1}, \lambda_{2}, \ldots, \lambda_{n}\right)$. Therefore, the columns of $Q$ are eigenvectors of $A(\Phi)$. Now, it is easy to see that

$$
\mathcal{E}_{\Phi}\left(v_{i}\right)=\sum_{j=1}^{n} Q_{i j}\left|\lambda_{j}\right|, \text { for } i=1,2, \ldots n
$$

where $Q_{i j}=\left|q_{i j}\right|^{2}$.

Let $\mathbb{C}_{n \times n}$ denote the set of all $n \times n$ complex matrices. Consider the function $\Omega_{i}$ : $\mathbb{C}_{n \times n} \rightarrow \mathbb{C}$ such that $\Omega_{i}(B)=b_{i, i}$, for $i=1,2, \ldots, n$, where $b_{i, i}$ is the $(i, i)$-th entry of $B$. Let $\Phi=(G, \varphi)$ be a $\mathbb{T}$-gain graph on $n$ vertices with adjacency matrix $A(\Phi)$. Then, $\Omega_{i}(|A(\Phi)|)=\mathcal{E}_{\Phi}\left(v_{i}\right), i=1,2, \ldots, n$. Now, it is clear that, for any two complex matrices $B$ and $C,\left|\Omega_{i}(B C)\right| \leq \Omega_{i}(|B C|)$. Since $\Omega_{i}$ is a positive linear functional, so the Hölder inequality holds, see [1]. That is, if $0<s, t \leq \infty$ with $1=\frac{1}{s}+\frac{1}{t}$, then

$$
\Omega_{i}(|B C|) \leq \Omega_{i}\left(|B|^{s}\right)^{\frac{1}{s}} \Omega_{i}\left(|C|^{t}\right)^{\frac{1}{t}}
$$

Lemma 3.2. Let $\Phi=(G, \varphi)$ be a $\mathbb{T}$-gain graph on $G$ of $n$ vertices and at least one edge. If $r \geq 2,0<s, t<\infty$ such that $\frac{1}{s}+\frac{1}{t}=1$, then

$$
\frac{\left(\Omega_{p}\left(|A(\Phi)|^{r}\right)\right)^{t}}{\left(\Omega_{p}\left(|A(\Phi)|^{s(r-1)+1}\right)\right)^{\frac{t}{s}}} \leq \mathcal{E}_{\Phi}\left(v_{p}\right), \quad p=1,2, \ldots, n
$$

Proof. Let $B=|A(\Phi)|^{r-\frac{1}{t}}$ and $C=|A(\Phi)|^{\frac{1}{t}}$. Then, by the Hölder inequality (7), we have

$$
\Omega_{p}\left(|A(\Phi)|^{r}\right)=\Omega_{p}\left(|A(\Phi)|^{r-\frac{1}{t}}|A(\Phi)|^{-\frac{1}{t}}\right) \leq\left(\Omega_{p}\left(|A(\Phi)|^{s r-\frac{s}{t}}\right)^{\frac{1}{s}}\right) \Omega_{p}(|A(\Phi)|)^{\frac{1}{t}}
$$

That is,

$$
\left(\Omega_{p}\left(|A(\Phi)|^{r}\right)\right)^{t} \leq\left(\Omega_{p}\left(|A(\Phi)|^{s r-\frac{s}{t}}\right)^{\frac{t}{s}}\right) \Omega_{p}(|A(\Phi)|)
$$

Since $0<s, t<\infty$, and $\frac{1}{s}+\frac{1}{t}=1$, so $r s-\frac{s}{t}=s(r-1)+1$. Therefore,

$$
\frac{\left(\Omega_{p}\left(|A(\Phi)|^{r}\right)\right)^{t}}{\left(\Omega_{p}\left(|A(\Phi)|^{s(r-1)+1}\right)\right)^{\frac{t}{s}}} \leq \Omega_{p}(|A(\Phi)|)=\mathcal{E}_{\Phi}\left(v_{p}\right), \quad p=1,2 \cdots, n
$$


Let $M_{k}(\Phi, p)$ denote the sum of the gains of directed $k$-walk from the vertex $v_{p}$ to itself, in the $\mathbb{T}$-gain graph $\Phi$. In the next result, we establish a bound of the vertex energy $\mathcal{E}_{\Phi}\left(v_{p}\right)$ in terms of $M_{k}(\Phi, p)$ and the vertex degree .

Lemma 3.3. Let $\Phi=(G, \varphi)$ be any $\mathbb{T}$-gain graph of $n$ vertices with at least one edge. Then

$$
\frac{d_{p}^{\frac{3}{2}}}{M_{4}(\Phi, p)^{\frac{1}{2}}} \leq \mathcal{E}_{\Phi}\left(v_{p}\right), \text { for all } p=1,2, \ldots, n
$$

Proof. In the Inequality (8), we substitute $r=2, s=3$ and $t=\frac{3}{2}$. Then we get

$$
\frac{\Omega_{p}\left(A(\Phi)^{2}\right)^{\frac{3}{2}}}{\Omega_{p}\left(A(\Phi)^{4}\right)^{\frac{1}{2}}} \leq \mathcal{E}_{\Phi}\left(v_{p}\right), \quad p=1,2, \ldots, n .
$$

Since $\Omega_{p}\left(A(\Phi)^{4}\right)=M_{4}(\Phi, p)$ and $\Omega_{p}\left(A(\Phi)^{2}\right)=d_{p}$, So the corollary follows.

Now, we establish a bound for $\mathcal{E}_{\Phi}\left(v_{j}\right)$ for $\mathbb{T}$-gain graph in terms of vertex degree of $v_{j}$ and the largest vertex degree $\Delta(G)$. For undirected graph $G$, these results are presented in [1].

Theorem 3.1. Let $\Phi=(G, \varphi)$ be any connected $\mathbb{T}$-gain graph with at least one edge. Then

$$
\mathcal{E}_{\Phi}\left(v_{j}\right) \geq \sqrt{\frac{d_{j}}{\Delta(G)}}, \quad \text { for all } v_{j} \in V(G) .
$$

Equality holds if and only if $\Phi \sim\left(K_{d_{j}, \Delta(G)}, 1\right)$.

Proof. Let $V(G)=\left\{v_{1}, v_{2}, \ldots, v_{n}\right\}$ be the vertex set of $G$. Let the degree of the vertex $v_{j}$ be $d_{j}$. Set $d_{j}=d$. Then the following three types of directed 4 -walks, starting from the vertex $v_{j}$ to itself, are possible:

1. $v_{j} \rightarrow v_{i} \rightarrow v_{j} \rightarrow v_{k} \rightarrow v_{j}$

2. $v_{j} \rightarrow v_{i} \rightarrow v_{s} \rightarrow v_{i} \rightarrow v_{j}$, where $v_{j} \neq v_{s}$;

3. $v_{j} \rightarrow v_{i} \rightarrow v_{s} \rightarrow v_{k} \rightarrow v_{j}$, where four vertices are mutually distinct.

Now the maximum value of the sum of the gains of the walks of type 1 is $d^{2}$. Similarly, for the type 2, the maximum value is $d(\Delta(G)-1)$, and for the type 3 , the maximum value is $2 \sum_{t=1}^{p} \cos \left(\theta_{t}\right)$, where $p \leq \frac{d(\Delta(G)-1)(d-1)}{2}$ and $\varphi\left(\overrightarrow{C_{m}}\right)=e^{i \theta_{m}}, \overrightarrow{C_{m}}$ is a 4-cycle formed by this 
walk. Thus the maximum value is $d(\Delta(G)-1)(d-1)$, and hence $M_{4}(\Phi, j) \leq d^{2} \Delta(G)$. Now, by Lemma 3.3. we have $\mathcal{E}_{\Phi}\left(v_{j}\right) \geq \sqrt{\frac{d_{j}}{\Delta(G)}}$.

If equality occurs in $(9)$, then $M_{4}(\Phi, j)=d^{2} \Delta(G)$. Therefore, $G=K_{d, \Delta(G)}$. Again from the equality $M_{4}(\Phi, j)=d^{2} \Delta(G)$, we have $\varphi\left(\overrightarrow{C_{m}}\right)=1$, for all cycle passing through the vertex $v_{j}$. Thus, by the Lemma 4.1, $\Phi$ is balanced. Hence $\Phi \sim\left(K_{d, \Delta(G)}, 1\right)$. Converse is easy to verify.

Corollary 3.1. Let $\Phi=(G, \varphi)$ be any connected $\mathbb{T}$-gain graph on a r-regular graph $G$. Then,

$$
\mathcal{E}_{\Phi}\left(v_{i}\right) \geq 1, \text { for all } v_{i} \in V(G) .
$$

Equality occurs if and only if $\Phi=\left(K_{r, r}, 1\right)$.

Proof. Since $G$ is a connected $r$-regular graph with vertex set $V(G)=\left\{v_{1}, v_{2}, \ldots, v_{n}\right\}$, so the degree of each vertex is same. For $i=1,2, \cdots, n, d_{i}=r=\Delta(G)$. Then by the Theorem 3.1. we have $\mathcal{E}_{\Phi}\left(v_{i}\right) \geq 1$, for all $v_{i} \in V(G)$. Equality occur if and only if $\Phi \sim\left(K_{r, r}, 1\right)$

In the next lemma, we show that the energy of a vertex is invariant under the switching equivalence of $\mathbb{T}$-gain graphs.

Lemma 3.4. Let $\Phi_{1}$ and $\Phi_{2}$ be any two switching equivalent $\mathbb{T}$-gain graphs on a graph $G$ with the vertex set $V(G)=\left\{v_{1}, v_{2}, \ldots, v_{n}\right\}$. Then for each $i$,

$$
\mathcal{E}_{\Phi_{1}}\left(v_{i}\right)=\mathcal{E}_{\Phi_{2}}\left(v_{i}\right)
$$

Proof. Since $\Phi_{1} \sim \Phi_{2}$, so $\operatorname{spec}\left(\Phi_{1}\right)=\operatorname{spec}\left(\Phi_{2}\right)$ and there is a diagonal unitary matrix $U$ such that $A\left(\Phi_{1}\right)=U A\left(\Phi_{2}\right) U^{*}$. Hence, by the Lemma 3.1, we have $\mathcal{E}_{\Phi_{1}}\left(v_{i}\right)=\mathcal{E}_{\Phi_{2}}\left(v_{i}\right)$ for each $i$.

In the next lemma, we provide a sufficient condition for the vertex energy of a $\mathbb{T}$-gain graph equals to the vertex energy of its underlying graph.

Lemma 3.5. Let $\Phi=(G, \varphi)$ be any $\mathbb{T}$-gain graph such that either $\Phi$ is balanced or $-\Phi$ is balanced. Then $\mathcal{E}_{\Phi}\left(v_{i}\right)=\mathcal{E}_{-\Phi}\left(v_{i}\right)=\mathcal{E}_{G}\left(v_{i}\right)$.

Proof. If $\Phi$ is balanced, then $\Phi \sim G$. Thus by the Lemma 3.4. $\mathcal{E}_{\Phi}\left(v_{i}\right)=\mathcal{E}_{G}\left(v_{i}\right)$, for $i=$ $1,2, \ldots, n$. Let $\left\{\lambda_{1}, \lambda_{2}, \cdots, \lambda_{n}\right\}$ be the spectrum of $\Phi$. Let $D=\operatorname{diag}\left(\lambda_{1}, \lambda_{2}, \cdots, \lambda_{n}\right)$. Then there exist an unitary matrix $Q$ such that $A(\Phi)=Q D Q^{*}$. Thus $A(-\Phi)=-A(\Phi)=$ $Q(-D) Q^{*}$. Therefore, by the Lemma 3.1, $\mathcal{E}_{\Phi}\left(v_{i}\right)=\mathcal{E}_{-\Phi}\left(v_{i}\right)$, for $i=1,2, \ldots, n$. Thus $\mathcal{E}_{\Phi}\left(v_{i}\right)=\mathcal{E}_{-\Phi}\left(v_{i}\right)=\mathcal{E}_{G}\left(v_{i}\right)$. If $-\Phi$ is balanced then we can prove the statement similarly. 
In the next Theorem, we provide a lower bound for the vertex energy of a $\mathbb{T}$-gain graph in terms of the degree of the vertex and the maximum vertex degree of the underlying graph. Theorem 3.2. Let $\Phi=(G, \varphi)$ be any connected $\mathbb{T}$-gain graph with at least one edge. Then

$$
\mathcal{E}_{\Phi}\left(v_{i}\right) \geq \frac{d_{i}}{\Delta(G)}, \quad \text { for all } v_{i} \in V(G)
$$

Equality occurs if and only if $\Phi \sim\left(K_{d, d}, 1\right)$, for some $d$.

Proof. Let $\Phi=(G, \varphi)$ be any $\mathbb{T}$-gain graph on $G$. Let $\lambda_{n} \leq \lambda_{n-1} \leq \cdots \leq \lambda_{1}$ be the eigenvalues of $\Phi$. By Theorem 2.1. $\max \left\{\lambda_{1},-\lambda_{n}\right\}=\rho(\Phi) \leq \rho(G) \leq \Delta(G)$. Hence $\lambda_{i} \in$ $[-\Delta(G), \Delta(G)]$ for all $i$. Therefore, $\left|\frac{\lambda_{i}}{\Delta(G)}\right| \leq 1$. Then $\left|\frac{\lambda_{i}}{\Delta(G)}\right| \geq\left(\frac{\lambda_{i}}{\Delta(G)}\right)^{2}$ and equality occur if and only if $\lambda_{i} \in\{-\Delta(G), 0, \Delta(G)\}$. Using Lemma 3.1, we have

$$
\mathcal{E}_{\Phi}\left(v_{i}\right)=\sum_{j=1}^{n}\left|q_{i j}\right|^{2}\left|\lambda_{j}\right| \geq \sum_{j=1}^{n}\left|q_{i j}\right|^{2} \frac{\lambda_{j}^{2}}{\Delta(G)}=\frac{d_{i}}{\Delta(G)}, \text { for all } v_{i} \in V(G),
$$

where $Q=\left(q_{i j}\right)$ is the unitary matrix whose columns are eigenvectors of $\Phi$. Since $G$ has at least one edge so there is a vertex $v_{j}$ such that $\mathcal{E}_{\Phi}\left(v_{j}\right)>0$. Therefore, if equality occur then either $\Delta(G)$ or $-\Delta(G)$ must be an eigenvalue of $\Phi$.

Now $\Delta(G)=\rho(\Phi) \leq \rho(G) \leq \Delta(G)$, so $\rho(G)=\rho(\Phi)$. Thus, by Theorem 2.1, either $\Phi$ is balanced or $-\Phi$ is balanced.

Case-I: If $\Phi$ is balanced, then by Lemma 3.4. $\mathcal{E}_{\Phi}\left(v_{i}\right)=\mathcal{E}_{G}\left(v_{i}\right)$ for all $v_{i} \in V(G)$. Then $\mathcal{E}_{G}\left(v_{i}\right)=\frac{d_{i}}{\Delta(G)}$. Therefore, by Lemma 2.5. $G$ is isomorphic to $K_{d, d}$, for some $d$. Hence $\Phi \sim\left(K_{d, d}, 1\right)$.

Case-II: If $-\Phi$ is balanced, then, similar to case-I, $-\Phi \sim\left(K_{d, d}, 1\right)$. Since the underlying graph is bipartite and $-\Phi$ is balanced, so, by Theorem 2.2 , $\Phi$ is balanced. Thus $\Phi \sim$ $\left(K_{d, d}, 1\right)$.

Using the above theorem, we prove that the energy of the complete bipartite $\mathbb{T}$-gain graph $K_{n, n}$ is always greater than or equal to the energy of the underlying graph.

Theorem 3.3. If $\Phi=\left(K_{n, n}, \varphi\right)$ is any $\mathbb{T}$-gain graph on the complete bipartite graph $K_{n, n}$, then $\mathcal{E}(\Phi) \geq \mathcal{E}\left(K_{n, n}\right)=2 n$, and equality holds if and only if $\Phi \sim\left(K_{n, n}, 1\right)$.

Proof. Let $V(G)=\left\{v_{1}, v_{2}, \ldots, v_{2 n}\right\}$ be the set of vertices of $\Phi$. Then, $\mathcal{E}(\Phi)=\mathcal{E}_{\Phi}\left(v_{1}\right)+$ $\mathcal{E}_{\Phi}\left(v_{2}\right)+\cdots+\mathcal{E}_{\Phi}\left(v_{2 n}\right)$. By Theorem 3.2 , we have

$$
\mathcal{E}(\Phi)=\sum_{j=1}^{2 n} \mathcal{E}_{\Phi}\left(v_{j}\right) \geq \sum_{j=1}^{2 n} \frac{d_{j}}{\Delta(G)}=2 n=\mathcal{E}\left(K_{n, n}\right) .
$$


It is easy to see that, equality occur if and only if $\Phi$ is balanced.

If $G$ is a $r$-regular graph of $n$ vertices, then $\mathcal{E}(G) \geq n$ and equality occur if and only if each component of $G$ is $K_{r, r}$ [Theorem 2.6. Next corollary is an extension of the above result for the $\mathbb{T}$-gain graph.

Corollary 3.2. Let $\Phi=(G, \varphi)$ be any $r$-regular $\mathbb{T}$-gain graph on $n$ vertices, where $r>0$. Then $\mathcal{E}(\Phi) \geq n$ and equality occur if and only if each component of $\Phi$ is switching equivalent to $\left(K_{r, r}, 1\right)$.

Proof. Let $G_{1}, G_{2}, \ldots, G_{k}$ be the connected components of $G$. Then $\mathcal{E}(\Phi)=\sum_{j=1}^{k} \mathcal{E}\left(\left(G_{j}, \varphi\right)\right)$. By the definition 3.1 and the equation $(6), \mathcal{E}\left(\left(G_{j}, \varphi\right)\right)=\sum_{v \in V\left(G_{j}\right)} \mathcal{E}_{\left(G_{j}, \varphi\right)}(v)$, for each $j=$ $1,2, \ldots, k$. Now, by Corollary 3.1. $\mathcal{E}_{\left(G_{j}, \varphi\right)}(v) \geq 1$, for any $v \in V\left(G_{j}\right)$. Then $\mathcal{E}\left(G_{j}, \varphi\right) \geq$ $\left|V\left(G_{j}\right)\right|$, for all $j=1,2, \ldots, k$. Thus $\mathcal{E}(\Phi) \geq n$. If $\mathcal{E}(\Phi)=n$, then $\mathcal{E}_{\Phi}(u)=1$, for all $u \in V(G)$. Therefore, by Corollary 3.1, each component of $\Phi$ is switching equivalent to $\left(K_{r, r}, 1\right)$.

Let $G$ be a semiregular bipartite graph with parameter $\left(n_{a}, n_{b}, r_{a}, r_{b}\right)$. A semi regular bipartite $\mathbb{T}$-gain graph with parameter $\left(n_{a}, n_{b}, r_{a}, r_{b}\right)$ is a $\mathbb{T}$-gain graph whose underlying graph is a semiregular bipartite graph of parameter $\left(n_{a}, n_{b}, r_{a}, r_{b}\right)$. The next bound is the generalization of a Theorem 2.7 for the $\mathbb{T}$-gain graphs.

Corollary 3.3. Let $\Phi=(G, \varphi)$ be any semiregular bipartite $\mathbb{T}$-gain graph with parameter $\left(n_{a}, n_{b}, r_{a}, r_{b}\right)$. Then $\mathcal{E}(\Phi) \geq n_{a} \sqrt{\frac{r_{a}}{r_{b}}}+n_{b} \sqrt{\frac{r_{b}}{r_{a}}}$. Equality occur if and only if each component is switching equivalent to $\left(K_{r_{a}, r_{b}}, 1\right)$.

Proof. Let $G_{1}, G_{2}, \ldots, G_{k}$ be the connected components of $G$. Then each $G_{j}$ is connected semiregular bipartite graph. Now, by applying Theorem 3.1 to each component $\left(G_{j}, \varphi\right)$, we get the result.

Remark 3.1. Hermitian adjacency matrices of mixed graphs are particular case of adjacency matrices of $\mathbb{T}$-gain graphs. Therefore all the above results for energy of a vertex holds true for mixed graphs.

\section{$4 \quad$ Lower bounds of energy of $\mathbb{T}$-gain graphs}

In this section, we establish several lower bounds for the energy of $\mathbb{T}$-gain graphs. We begin this section with the following theorem which gives a lower bound for the energy of a $\mathbb{T}$-gain graph in terms of the gain of the real parts of the fundamental cycles. 
Theorem 4.1. Let $\Phi=(G, \varphi)$ be any connected $\mathbb{T}$-gain graph on $n$ vertices. Let $T$ be a normal spanning tree of $G$, and $\left\{C_{1}, C_{2}, \ldots, C_{l}\right\}$ be the collection of all fundamental cycles in $G$ with respect to $T$. Then,

$$
\left.\mathcal{E}(\Phi) \geq 2 \sum_{j=1}^{l} \operatorname{Re}\left(\varphi\left(C_{j}\right)\right)\right)+\left(5 n-n^{2}-4\right) .
$$

The inequality is sharp.

Proof. Let $\Phi=(G, \varphi)$ be any connected $\mathbb{T}$-gain graph. Let $T$ be a normal spanning tree of $G$, and $\left\{C_{1}, C_{2}, \ldots, C_{l}\right\}$ be the collection of all fundamental cycles in $G$ with respect to $T$. Define a new $\mathbb{T}$-gain graph $\Phi^{\prime}$ on $G$ such that $\varphi^{\prime}(\vec{e})=1$ for all $e \in E(T)$ and $\varphi\left(C_{i}\right)=\varphi^{\prime}\left(C_{i}\right)$ for all $i$. So, by Lemma 2.2 , the $\mathbb{T}$-gain graphs $\Phi$ and $\Phi^{\prime}$ are switching equivalent. Therefore,

$$
\sum_{i, j} \varphi^{\prime}\left(\overrightarrow{e_{i, j}}\right)=\sum_{j=1}^{l}\left\{\varphi^{\prime}\left(\vec{C}_{j}\right)+\varphi^{\prime}\left(\vec{C}_{j}\right)^{-1}\right\}+2(n-1)=2 \sum_{j=1}^{l} \operatorname{Re}\left(\varphi\left(C_{j}\right)\right)+2(n-1) .
$$

Now, $\operatorname{Re}\left(\operatorname{trace}\left(A\left(K_{n}\right) A\left(\Phi^{\prime}\right)\right)\right)=\sum_{i, j} \varphi^{\prime}\left(\overrightarrow{e_{i, j}}\right)$. Let $\left|\lambda_{1}\right| \geq\left|\lambda_{2}\right| \geq \cdots \geq\left|\lambda_{n}\right|$ be the singular values of $\Phi^{\prime}$. Since $\operatorname{spec}\left(K_{n}\right)=\left\{-1^{(n-1)},(n-1)^{(1)}\right\}$, by Theorem 2.8, we have $\operatorname{Re}\left(\operatorname{trace}\left(A\left(K_{n}\right) A\left(\Phi^{\prime}\right)\right) \leq(n-1)\left|\lambda_{1}\right|+\sum_{j=2}^{n-1}\left|\lambda_{j}\right|\right.$, and hence $\sum_{i, j} \varphi^{\prime}\left(\overrightarrow{e_{i, j}}\right) \leq(n-2)\left|\lambda_{1}\right|+\mathcal{E}\left(\Phi^{\prime}\right)$. So, by equation 11 , we have $2 \sum_{j=1}^{l} \operatorname{Re}\left(\varphi\left(C_{j}\right)\right)+2(n-1) \leq(n-2)\left|\lambda_{1}\right|+\mathcal{E}\left(\Phi^{\prime}\right)$. Now, using Theorem 2.1, we get $\left|\lambda_{1}\right|=\rho\left(\Phi^{\prime}\right)=\rho(\Phi) \leq \rho(G) \leq \Delta \leq(n-1)$. Thus

$$
\sum_{j=1}^{l} \operatorname{Re}\left(\varphi\left(C_{j}\right)\right)+2(n-1) \leq(n-2)(n-1)+\mathcal{E}(\Phi),
$$

and hence

$$
\mathcal{E}(\Phi) \geq 2 \sum_{j=1}^{l} \operatorname{Re}\left(\varphi\left(C_{j}\right)\right)+\left(5 n-n^{2}-4\right)
$$

Now, if $\Phi \sim\left(K_{n}, 1\right)$, then $2 \sum_{j=1}^{l} \operatorname{Re}\left(\varphi\left(C_{j}\right)\right)=n^{2}-3 n+2$ and hence equality holds in equation (10).

If $G$ is a complete bipartite graph, then $A(G)$ has exactly one positive eigenvalue. Also, if $G$ is any non-complete bipartite graph on more than 4 vertices, then it contains $P_{4}$ as an induced subgraph, and hence $A(G)$ has at least two positive eigenvalues. So, if $G$ is a bipartite graph on more than 4 vertices, then $G$ is complete bipartite if and only if $A(G)$ 
has exactly one positive eigenvalue. Our next objective is to study the counter part of this property for the $\mathbb{T}$-gain graphs. The following lemma is a key in the proof of Theorem 4.2 . This gives a sufficient condition a $\mathbb{T}$-gain graph to be balanced.

Lemma 4.1. Let $\Phi=(G, \varphi)$ be any $\mathbb{T}$-gain graph on a complete bipartite graph $G$. If every 4-cycle which passes through the vertex $v$, for some vertex $v$ of $G$, has gain 1 , then $\Phi$ is balanced.

Proof. Let $\Phi=(G, \varphi)$ be any $\mathbb{T}$-gain graph on a complete bipartite graph $G$. Let $v$ be a vertex in $G$ such that the gain of any 4-cycle passing through the vertex $v$ is 1 . First let us show that gain of any four cycle in $G$ is 1 . Let $C_{4} \equiv v_{1}-v_{2}-v_{3}-v_{4}-v_{1}$ be any 4-cycle in $\Phi$ such that $C_{4}$ does not contain the vertex $v$. Without loss of generality, let us assume that $v_{2} \sim v$. Now, consider the two 4-cycles: $C_{4}(v) \equiv v_{1}-v_{2}-v-v_{4}-v_{1}$ and $C_{4}^{\prime}(v) \equiv v-v_{2}-v_{3}-v_{4}-v$. Then $\varphi\left(\overrightarrow{C_{4}}\right)=\varphi\left(\overrightarrow{C_{4}(v)}\right) \varphi\left(\overrightarrow{C_{4}^{\prime}(v)}\right)=1$.

Let $C_{2 p}$ be any cycle in $G$ on $2 p$ vertices. Without loss of generality, let us assume that $C_{2 p} \equiv v_{1}-v_{2}-v_{3}-v_{4}-\cdots-v_{(2 p-1)}-v_{2 p}-v_{1}$. Then

$$
\begin{aligned}
\varphi\left(\overrightarrow{C_{2 p}}\right)= & \varphi\left(\overrightarrow{e_{1,2}}\right) \varphi\left(\overrightarrow{e_{2,3}}\right) \varphi\left(\overrightarrow{e_{3,4}}\right) \ldots \varphi\left(\overrightarrow{e_{(2 p-1), 2 p}}\right) \varphi\left(\overrightarrow{e_{2 p, 1}}\right) \\
= & \left\{\varphi\left(\overrightarrow{e_{1,2}}\right) \varphi\left(\overrightarrow{e_{2,3}}\right) \varphi\left(\overrightarrow{e_{3,4}}\right) \varphi\left(\overrightarrow{e_{4,1}}\right)\right\} \\
& \left\{\varphi\left(\overrightarrow{e_{1,4}}\right) \varphi\left(\overrightarrow{e_{4,5}}\right) \varphi\left(\overrightarrow{e_{5,6}}\right) \varphi\left(\overrightarrow{e_{6,1}}\right)\right\} \\
& \vdots \\
& \left\{\varphi\left(\overrightarrow{e_{1,(2 p-2)}}\right) \varphi\left(\overrightarrow{e_{(2 p-2),(2 p-1)}}\right) \varphi\left(\overrightarrow{e_{(2 p-1), 2 p}}\right) \varphi\left(\overrightarrow{e_{2 p, 1}}\right)\right\} \\
= & 1 .
\end{aligned}
$$

Thus $\Phi$ is balanced.

Theorem 4.2. Let $\Phi=(G, \varphi)$ be any $\mathbb{T}$-gain graph on a connected bipartite graph $G$. Then $\Phi$ has exactly one positive eigenvalue if and only if $\Phi$ is a balanced complete bipartite graph.

Proof. Let $\Phi=(G, \varphi)$ have exactly one positive eigenvalue. If the number of vertices of $G$ is two or three, then $G$ must be $K_{2}$ or $K_{1,2}$, respectively. Therefore, in both the cases, $\Phi$ is a balanced compete bipartite graph. Now we consider a graph $G$ with $|V(G)| \geq 4$. Suppose that $P_{4}$ is an induced subgraph of $G$. So $\left(P_{4}, \varphi\right)$ is an induced $\mathbb{T}$-gain subgraph of $\Phi$. As $P_{4}$ is a tree, so the spectrum of $P_{4}$ with respect to $\varphi$ is same as that of $\operatorname{spec}\left(P_{4}\right)$. Thus $P_{4}$ has two positive eigenvalue with respect to $\varphi$. Therefore, by the interlacing theorem, $\Phi$ has at least two positive eigenvalue, a contradiction. Thus $G$ can not have $P_{4}$ as an induced subgraph, 
and hence the diameter of $G$ is at most 2. Now it is easy to see that any two non adjacent vertices have the same neighbors. Thus $G$ is complete multipartite. But $G$ is bipartite, so $G$ is complete bipartite.

Now we consider the following two cases to show that $\Phi$ is balanced.

Case 1: If $G$ does not contain any cycles, then $G$ must be a star, and hence $\Phi$ is balanced. Case 2: If $G$ contains cycles, then it must contains an induced $C_{4}$. Let $\varphi\left(\overrightarrow{C_{4}}\right)=e^{i \theta}, \theta \in$ $[0,2 \pi)$.

Let $C=\left(C_{4}, \varphi\right)$ be an induced subgraph of $\Phi$ whose underlying graph is $C_{4}$. Therefore, by Theorem 2.3, we have

$$
\operatorname{spec}(C)=\left\{2 \cos \left(\frac{\theta}{4}\right), 2 \cos \left(\frac{\theta}{4}+\frac{\pi}{2}\right), 2 \cos \left(\frac{\theta}{4}+\pi\right), 2 \cos \left(\frac{\theta}{4}+\frac{3 \pi}{2}\right)\right\}
$$

Let $x=\frac{\theta}{4} \in\left[0, \frac{\pi}{2}\right)$. It is easy to see that $\operatorname{spec}(C)$ has two positive and two negative eigenvalues if and only if $x \in\left(0, \frac{\pi}{2}\right)$. Hence $\operatorname{spec}(C)$ has exactly one positive eigenvalue if and only if $x=0$. Now, by interlacing theorem, $\Phi$ cannot have any induced 4-cycle $C_{4}$ such that $\varphi\left(\overrightarrow{C_{4}}\right)=e^{i \theta}$, where $\theta \in(0,2 \pi)$. Therefore, for any induced 4-cycle $C$ in $G$, we have $\varphi(C)=1$. Thus, by Lemma 4.1. $\Phi$ is balanced. Conversely, if $\Phi$ is a balanced complete bipartite $\mathbb{T}$-gain graph, then $\Phi$ has exactly one positive eigenvalue.

Next result gives a lower bound of energy of $\mathbb{T}$-gain graph in terms of spectral radius.

Theorem 4.3. If $\Phi=(G, \varphi)$ be any $\mathbb{T}$-gain graph on a connected graph $G$. Then $\mathcal{E}(\Phi) \geq$ $2 \rho(\Phi)$. If $G$ is bipartite then equality occurs if and only if $\Phi \sim\left(K_{p, q}, 1\right)$ for some $p, q$.

Proof. Let $\left\{\lambda_{1}, \lambda_{2}, \ldots, \lambda_{n}\right\}$ be the spectrum of $\Phi$ such that $\lambda_{1} \geq \lambda_{2} \geq \cdots \geq \lambda_{n}$. Now $\lambda_{1}+\lambda_{2}+\cdots+\lambda_{n}=0$. Therefore, $2\left|\lambda_{1}\right| \leq\left|\lambda_{1}\right|+\left|\lambda_{2}\right|+\cdots+\left|\lambda_{n}\right|$. Thus $\mathcal{E}(\Phi) \geq 2 \rho(\Phi)$.

Let $G$ be bipartite. Since $\left|\lambda_{1}\right|=\left|\lambda_{2}\right|+\cdots+\left|\lambda_{n}\right|$ holds if and only if all of $\lambda_{j}^{\prime} s$, for $j=$ $2,3, \ldots, n$ are of the same sign. Therefore, equality occur if and only if $\Phi$ has only one positive eigenvalue. Hence, by the Theorem 4.2 , equality holds if and only if $\Phi \sim\left(K_{p, q}, 1\right)$.

Let $J$ denote the all 1's matrix of appropriate size. The following two theorems provide a lower bound for energy of $\mathbb{T}$-gain graph in terms of the number of vertices and the gains of fundamental cycles.

Theorem 4.4. Let $\Phi=(G, \varphi)$ be any connected $\mathbb{T}$-gain graph with $n$ vertices and $\left\{C_{1}, C_{2}, \ldots, C_{l}\right\}$ be the collection of all fundamental cycles in $G$ with respect to a normal spanning tree $T$. Then

$$
\mathcal{E}(\Phi) \geq 4+\frac{4}{n}\left\{\sum_{j=1}^{l} \operatorname{Re}\left(\varphi\left(C_{j}\right)\right)-1\right\}
$$


The inequality is sharp.

Proof. Let $\left\{\lambda_{1}, \lambda_{2}, \ldots, \lambda_{n}\right\}$ be the spectrum of $\Phi$ such that $\left|\lambda_{1}\right| \geq\left|\lambda_{2}\right| \geq \cdots \geq\left|\lambda_{n}\right|$. Define a new $\mathbb{T}$-gain $\Phi^{\prime}$ on $G$ such that $\varphi^{\prime}(\vec{e})=1$ for all $e \in E(T)$ and $\varphi\left(C_{i}\right)=\varphi^{\prime}\left(C_{i}\right)$ for all $i$. So, by Lemma 2.2 , the $\mathbb{T}$-gain graphs $\Phi$ and $\Phi^{\prime}$ are switching equivalent. Then $\sum_{i, j} \varphi^{\prime}\left(\overrightarrow{e_{i j}}\right)=2 \sum_{j=1}^{l} \operatorname{Re}\left(\varphi\left(C_{j}\right)\right)+2(n-1)$. By Theorem 2.8 , we have

$$
\operatorname{Re}(\operatorname{trace}(A(\Phi) J)) \leq n\left|\lambda_{1}\right|,
$$

and hence

$$
2(n-1)+2 \sum_{j=1}^{l} \operatorname{Re}\left(\varphi\left(C_{j}\right)\right) \leq n\left|\lambda_{1}\right| .
$$

As $\left|\lambda_{1}\right| \leq\left|\lambda_{2}\right|+\cdots+\left|\lambda_{n}\right|$, so $\left|\lambda_{1}\right| \leq \frac{\mathcal{E}(\Phi)}{2}$. Therefore,

$$
\mathcal{E}(\Phi) \geq 4+\frac{4}{n}\left\{\sum_{j=1}^{l} \operatorname{Re}\left(\varphi\left(C_{j}\right)\right)-1\right\} .
$$

Let us take $\Phi \sim(G, 1)$, where $G=K_{r, r, \cdots, r}$ is a connected complete $p$-partite graph on $m$ edges and $n$ vertices. Then the right hand side expression $12 p$ becomes $4+\frac{4}{n}(m-n+1-1)$, which is $\frac{4 m}{n}$. Since $G$ is a complete multipartite graph, $G$ has exactly one positive eigenvalue. Thus, if $\lambda_{1}, \ldots, \lambda_{n}$ are the eigenvalues of $\Phi$, then $\left|\lambda_{1}\right|=\left|\lambda_{2}\right|+\cdots+\left|\lambda_{n}\right|$, and hence $\mathcal{E}(\Phi)=$ $2\left|\lambda_{1}\right|=2 \lambda_{1}$. Also the spectral radius of $\Phi$ is $(r-1)$, the degree of each vertex in $G$, and the degree of each vertex of $G$ is $\frac{2 m}{n}$. Therefore, $\mathcal{E}(\Phi)=\frac{4 m}{n}$. Hence the inequality is sharp.

If the underlying graph is a bipartite graph, then we can completely characterize the classes for which equality holds in (12).

Corollary 4.1. Let $\Phi=(G, \varphi)$ be any connected $\mathbb{T}$-gain graph on a bipartite graph $G$ with $n$ vertices. Then

$$
\mathcal{E}(\Phi) \geq 4+\frac{4}{n}\left\{\sum_{j=1}^{l} \operatorname{Re}\left(\varphi\left(C_{j}\right)\right)-1\right\} .
$$

Equality occurs if and only if $\Phi \sim\left(K_{\frac{n}{2}, \frac{n}{2}}, 1\right)$.

Proof. Let $\Phi=(G, \varphi)$ be any connected $\mathbb{T}$-gain graph on a bipartite graph $G$ with $m$ edges and $n$ vertices. Let $\left\{\lambda_{1}, \lambda_{2}, \cdots, \lambda_{n}\right\}$ be the spectrum of $\Phi$ such that $\left|\lambda_{1}\right| \geq\left|\lambda_{2}\right| \cdots \geq\left|\lambda_{n}\right|$. Let $\left\{C_{1}, C_{2}, \cdots, C_{l}\right\}$ be the fundamental cycles of $G$ with respect to a normal spanning tree $T$. Now the inequality is clear from the Theorem 4.4. Let us consider the equality,

$$
\mathcal{E}(\Phi)=4+\frac{4}{n}\left\{\sum_{j=1}^{l} \operatorname{Re}\left(\varphi\left(C_{j}\right)\right)-1\right\} .
$$


Then from the proof of the Theorem 4.4, we have

$$
\mathcal{E}(\Phi) \geq 2\left|\lambda_{1}\right| \geq 4+\frac{4}{n}\left\{\sum_{j=1}^{l} \operatorname{Re}\left(\varphi\left(C_{j}\right)\right)-1\right\}
$$

Since $\Phi$ is a bipartite $\mathbb{T}$-gain graph, so $\Phi$ must satisfy the following equality.

(i) $\mathcal{E}(\Phi)=2\left|\lambda_{1}\right|=2 \rho(\Phi)$

(ii) $2 \lambda_{1}=2\left|\lambda_{1}\right|=4+\frac{4}{n}\left\{\sum_{j=1}^{l} \operatorname{Re}\left(\varphi\left(C_{j}\right)\right)-1\right\}$

Now by the Theorem 4.3 , the equation $(i)$ is satisfied if and only if $\Phi \sim\left(K_{p, q}, 1\right)$, for some $p$ and $q$. Then $\Phi$ is balanced, so from equation $(i i)$, we have $\lambda_{1}=\frac{2 m}{n}$. Since $\Phi \sim\left(K_{p, q}, 1\right)$, so $\lambda_{1}=\sqrt{p q}, n=p+q$ and $m=p q$. Therefore, we have $\sqrt{p q}=\frac{2 p q}{p+q}$. That is $p=q=\frac{n}{2}$. Thus $\Phi \sim\left(K_{\frac{n}{2}, \frac{n}{2}}, 1\right)$. Converse is easy to verify.

The following lemma is about the change in the energy of a graph obtained from a graph by removing a cut set. This will be useful in the proof of some of the following results.

Lemma 4.2. Let $\Phi=(G, \varphi)$ be a $\mathbb{T}$-gain graph and $E$ be a cut set of $\Phi$. Then $\mathcal{E}(\Phi-E) \leq$ $\mathcal{E}(\Phi)$.

Proof. For any cut set $E$ of $G$, there exist two induced sub graphs $L$ and $M$ complement to each other in $G$ such that $G-E=L \oplus M$. Then $\Phi-E=(L, \varphi) \oplus(M, \varphi)$. Now $A(\Phi)$ can be expressed as $\left[\begin{array}{cc}A((L, \varphi)) & X \\ X^{*} & A((M, \varphi))\end{array}\right]$. Therefore by the Theorem $2.10, \mathcal{E}(\Phi) \geq$ $\mathcal{E}(A(L, \varphi))+\mathcal{E}(A(M, \varphi))=\mathcal{E}(\Phi-E)$.

In the next result, we establish a connection between the gain energy and the matching number of a graph. This result is a counter part (for the $\mathbb{T}$-gain graphs) of Lemma 2.7 and Theorem 2.4 for undirected graph, a main result in [14] for skew energy of oriented graph, and Theorem 2.5 for mixed graph.

Theorem 4.5. Let $\Phi=(G, \varphi)$ be a $\mathbb{T}$-gain graph, and let $\mu(G)$ be the matching number of G. Then $\mathcal{E}(\Phi) \geq 2 \mu(G)$.

Proof. Let $\Phi=(G, \varphi)$ be any $\mathbb{T}$-gain graph with matching number $\mu(G)$. We prove the result by induction on $\mu(G)$. If $\mu(G)=0$, then $\mathcal{E}(\Phi)=2 \mu(G)=0$. If $\mu(G)=1$, then $G$ must be $K_{1, p}$, for some $p$, together with some isolated vertices. Therefore, $\Phi \sim(G, 1)$. Thus $\mathcal{E}(\Phi)=2 \sqrt{p} \geq 2=2 \mu(G)$. Let us assume that for any $\mathbb{T}$-gain graph $\Psi=(H, \psi)$ 
with matching number $\mu(H)<\mu(G), \mathcal{E}(\Psi) \geq 2 \mu(H)$. Let $M$ be a maximum matching of $G$ and $e \in M$. Now consider an induced subgraph $G-[e]$. Then $\mu(G-[e])=\mu(G)-1$. By induction, we have $\mathcal{E}((G-[e], \varphi)) \geq 2 \mu(G-[e])$. Let $E$ be the set of edges in $G$ which are incident with the edge $e$. Then $E$ is a cut set, and $(G-E)=(G-[e]) \oplus K_{2}$. By the Lemma 4.2, $\mathcal{E}(\Phi) \geq \mathcal{E}(\Phi-E)$. Now $\mathcal{E}(\Phi) \geq \mathcal{E}(\Phi-E)=\mathcal{E}((G-[e], \varphi))+\mathcal{E}\left(\left(K_{2}, \varphi\right)\right) \geq$ $2 \mu(G)-2+2=2 \mu(G)$. Hence the result.

We shall discuss the sharpness of the inequality in the above bound in Theorem 4.7 .

The following lemma is the counter part of Lemma 2.1 for the $\mathbb{T}$-gain graphs.

Lemma 4.3. Let $\Phi=(G, \varphi)$ be a $\mathbb{T}$-gain graph. If $E$ is a cut set in $G$ such that $V(G)=$ $V_{1} \cup V_{2}$ and all the edges of $E$ are from the vertices of $V_{1}$ to a fixed vertex of $V_{2}$, then $\mathcal{E}(\Phi-E)<\mathcal{E}(\Phi)$.

Proof. Let $E$ be a cut set, and $L$ and $M$ be two complementary induced subgraphs in $G$ corresponding to $E$. Let us assume that the edges of $E$ are incidence with a single vertex $v$ of $M$. After a suitable relabeling of vertices, we can express $A(\Phi)=\left[\begin{array}{cc}A((L, \varphi)) & X \\ X^{*} & A((M, \varphi))\end{array}\right]$ such that the first column of the matrix $X$, say $y$, corresponds to the vertex $v$. Hence all the entries of the matrix $X$ are zero, except the first column. Now, by Lemma 4.2 , $\mathcal{E}(\Phi-E) \leq \mathcal{E}(\Phi)$. Suppose that $\mathcal{E}(\Phi-E)=\mathcal{E}(\Phi)$. Then, by Theorem 2.10, there exists two unitary matrices $P$ and $Q$, such that $\left[\begin{array}{cc}P A((L, \varphi)) & P X \\ Q X^{*} & Q A((M, \varphi))\end{array}\right]$ is positive semi definite. As $(P X)^{*}=Q X^{*}$, we have $Q=\left[\begin{array}{cc}\beta & 0 \\ 0 & Q_{1}\end{array}\right]$ with $|\beta|=1$, and $Q_{1}$ is unitary matrix. Let $A((M, \varphi))=\left[\begin{array}{ll}0 & z^{*} \\ z & N\end{array}\right]$. Then $Q A((M, \varphi))=\left[\begin{array}{cc}0 & \beta z^{*} \\ Q_{1} z & Q_{1} N\end{array}\right]$ is positive semi definite. So $Q_{1} z=0$ and $\beta z^{*}=0$. That is $z=0$. Hence $A((M, \varphi))=\left[\begin{array}{cc}0 & 0 \\ 0 & N\end{array}\right]$. Therefore $A(\Phi)=$ $\left[\begin{array}{ccc}A((L, \varphi)) & y & 0 \\ y^{*} & 0 & 0 \\ 0 & 0 & N\end{array}\right]$. Now $\left.\mathcal{E}(A((L, \varphi)))+\mathcal{E}(N)=\mathcal{E}(A((L, \varphi)))+\mathcal{E}(A(M, \varphi))\right)=\mathcal{E}(\Phi-$ $E)=\mathcal{E}(\Phi)=\mathcal{E}\left(\left[\begin{array}{cc}A((L, \varphi)) & y \\ y^{*} & 0\end{array}\right]\right)+\mathcal{E}(N)$. That is $\mathcal{E}\left(\left[\begin{array}{cc}A((L, \varphi)) & y \\ y^{*} & 0\end{array}\right]\right)=\mathcal{E}(A((L, \varphi)))$. Hence, by Lemma 2.9, $y=0$. Thus $E$ is empty. Which is a contradiction.

The following lemma provides a (spectral) sufficient condition for a graph to have perfect matching. This is a counter part of Lemma 2.9 for the $\mathbb{T}$-gain graphs. 
Lemma 4.4. If $\Phi=(G, \varphi)$ is a connected $\mathbb{T}$-gain graph and $\mathcal{E}(\Phi)=2 \mu(G)$, then $G$ has a perfect matching

Proof. Suppose that $G$ has no perfect matching. Let $M$ be any maximum matching of $G$. Since $G$ is a connected graph, so there exist a vertex $u$ which is not adjacent with any edges in $M$. Then $\mu(G)=\mu(G-u)$. Let $K_{1}$ be the graph which is an isolated vertex $u$. Let $E$ be the set of all edges incident with the vertex $u$ in $G$. Then, $E$ is a cut set, and $\Phi-E=(\Phi-u) \oplus K_{1}$. Therefore, by Lemma 4.3 and Theorem 4.5, $\mathcal{E}(\Phi)>\mathcal{E}(\Phi-E)=$ $\mathcal{E}(\Phi-u)+0 \geq 2 \mu(G-u)=2 \mu(G)$. That is, $\mathcal{E}(\Phi)>2 \mu(G)$, a contradiction. Thus $G$ has a perfect matching.

Now, let us establish a couple of lemmas about the energy of a $\mathbb{T}$-gain graph in terms of the matching number of the underlying graph.

Lemma 4.5. Let $\Phi=(G, \varphi)$ be a connected $\mathbb{T}$-gain graph with a pendant vertex. If $G$ is not $K_{2}$, then $\mathcal{E}(\Phi)>2 \mu(G)$.

Proof. Let $v$ be a pendent vertex of $G, u$ be its unique neighbor vertex, and $e$ be the edge between them. Then the induced subgraphs $(G-[e])$ and $K_{2}$ are complement to each other in $G$. Let $E$ be the collection of all edges between the vertex $u$ and the vertices of $G-\{u, v\}$. Then $G-E=(G-[e]) \oplus K_{2}$. By Lemma 4.3, $\mathcal{E}(\Phi)>\mathcal{E}(\Phi-E)=\mathcal{E}((G-[e], \varphi))+\mathcal{E}\left(\left(K_{2}, \varphi\right)\right)$. Also $\mu(G-[e])=\mu(G)-1$. Therefore, by Theorem 4.5, $\mathcal{E}(\Phi)>2 \mu(G)$.

Lemma 4.6. Let $\Phi=(G, \varphi)$ be a connected $\mathbb{T}$-gain graph and $L$ be an induced subgraph of $G$. If $\mathcal{E}((L, \varphi))>2 \mu(L)$ and $\mu(G)=\mu(L)+\mu(G-L)$. Then $\mathcal{E}(\Phi)>2 \mu(G)$.

Proof. Since $L$ is an induced subgraph of $G$, so $(G-L)$ is the complementary induced subgraph of $G$. Let $E$ be a cut set of $G$ such that $(G-E)=(G-L) \oplus L$. Then $(\Phi-E)=$ $(G-L, \varphi) \oplus(L, \varphi)$. Since $E$ is a cut set of $G$, so, by Lemma 4.2, $\mathcal{E}(\Phi) \geq \mathcal{E}(\Phi-E)=$ $\mathcal{E}((L, \varphi))+\mathcal{E}((G-L, \varphi))$. Now, by Theorem 4.5 and the hypothesis, we have $\mathcal{E}(\Phi)>2 \mu(L)+$ $2 \mu(G-L)=2 \mu(G)$. Hence, $\mathcal{E}(\Phi)>2 \mu(G)$.

Lemma 4.7. Let $\Phi=(G, \varphi)$ be any $\mathbb{T}$-gain graph on a connected graph $G$ which is given in the figure 1 . Then $\mathcal{E}(\Phi)>2 \mu(G)$.

Proof. Let $E$ be the cut set consist of the set of edges which are incidence with the edge $e$ in Figure 1. Then $G-E=K_{2} \oplus P_{4}$. Now, by Lemma 4.2, $\mathcal{E}(\Phi) \geq \mathcal{E}(\Phi-E)=$ $\mathcal{E}\left(\left(K_{2}, \varphi\right)\right)+\mathcal{E}\left(\left(P_{4}, \varphi\right)\right)$. Since $\left(K_{2}, \varphi\right) \sim\left(K_{2}, 1\right)$ and $\left(P_{4}, \varphi\right) \sim\left(P_{4}, 1\right)$, so by the Lemma 4.5 , we have $\mathcal{E}(\Phi)>2+2 \mu\left(P_{4}\right)=2 \mu(G)$. 


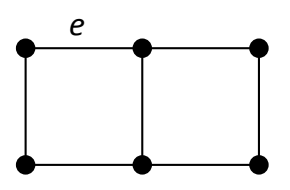

Figure 1: Graph $G$

In the next theorem, we characterize the class of bipartite $\mathbb{T}$-graphs for which equality holds in Theorem 4.5. Define $N(u)=\{x \in V(G): u \sim x\}$.

Theorem 4.6. Let $\Phi=(G, \varphi)$ be any connected $\mathbb{T}$-gain bipartite graph with $n$ vertices. Then $\mathcal{E}(\Phi)=2 \mu(G)$ if and only if $\Phi \sim\left(K_{\frac{n}{2}}, \frac{n}{2}, 1\right)$.

Proof. First let us show that $G$ is complete bipartite using induction on the number of vertices. Let $|V(G)|=2$. If $\mathcal{E}(\Phi)=2 \mu(G)$, then it is clear that $G=K_{1,1}$. Let us assume that for any connected bipartite $\mathbb{T}$-gain graph $(H, \psi)$ with $|V(H)|<n$, if $\mathcal{E}((H, \psi))=2 \mu(H)$, then $H$ is a complete bipartite graph with same partition size. Let $\Phi=(G, \varphi)$ be any connected bipartite $\mathbb{T}$-gain graph with $n$ vertices such that $\mathcal{E}(\Phi)=2 \mu(G)$. By the Lemma 4.4. $G$ has perfect matching, $M$ (say). Let $X$ and $Y$ be the vertex partition of $G$ such that $|X|=|Y|=\frac{n}{2}$.

Claim 1: For any vertex $u \in X, N(u)=Y$.

Suppose that $N(u)$ is a proper subset of $Y$. Let $v^{\prime} \in Y \backslash N(u)$. Then there exists vertices $u^{\prime} \in X$ and $v \in Y$ such that the edges $(u, v)$ and $\left(u^{\prime}, v^{\prime}\right)$ are in $M$.

Let $P$ be an induced subgraph formed by the vertices $\left\{u, v, u^{\prime}, v^{\prime}\right\}$. The vertices $u^{\prime}$ and $v$ are not adjacent in $G$. Suppose they are adjacent. Then $P$ is isomorphic to $P_{4}$. If $|V(G)|=4$, then, by Lemma 4.5, $\mathcal{E}(\Phi)>2 \mu(G)$, a contradiction. Thus $G=K_{2,2}$.

If $|V(G)|>4$, then it is clear that $\mu(G)=\mu(P)+\mu(G-P)$. By Lemma 4.5, $\mathcal{E}((P, \varphi))>$ $2 \mu(P)$. Then, by Lemma $4.6, \mathcal{E}(\Phi)>2 \mu(G)$, which is again a contradiction. Thus $u^{\prime} \nsim v$.

Let $Q=(G-P)$. Then $Q$ is the complementary induced subgraph of $P$ in $G$. Therefore, $\mu(G)=\mu(P)+\mu(Q)=2+\mu(Q)$. Now, we have $2 \mu(G)=\mathcal{E}(\Phi) \geq \mathcal{E}((P, \varphi))+\mathcal{E}((Q, \varphi)) \geq$ $2(2+\mu(Q))=2 \mu(G)$. Thus, $\mathcal{E}((Q, \varphi))=2 \mu(Q)$. Then, by induction hypothesis, $Q$ is complete bipartite graph with partition $X^{\prime}$ and $Y^{\prime}$ such that $\left|X^{\prime}\right|=\left|Y^{\prime}\right|$. Then $X=$ $X^{\prime} \cup\left\{u, u^{\prime}\right\}$ and $Y=Y^{\prime} \cup\left\{v, v^{\prime}\right\}$.

sub claim: For every $x \in X^{\prime}$ the vertices $x$ and $v$ are adjacent, and for every $y \in Y^{\prime}$ the vertices $y$ and $u$ are adjacent. 


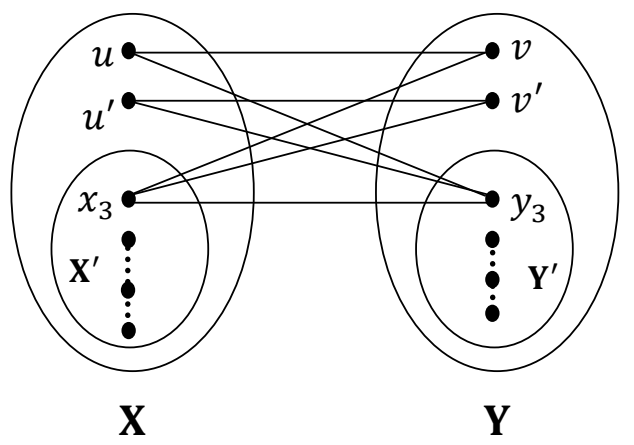

Figure 2: Graph $G$

Since $G$ is connected, so at least one of the vertices of $u$ or $v$ is adjacent with the vertices in $Y^{\prime}$ or $X^{\prime}$, respectively. Without loss of generalities, let us assume that $u \sim y$ for some $y \in Y^{\prime}$. Now, every vertex of $X^{\prime}$ is adjacent with $v$. Otherwise, there is a vertex $x \in X^{\prime}$ such that $x \nsim v$. Then the induced underlying subgraph $H_{1}$ (say) formed by the vertices $\{u, v, y, x\}$ is isomorphic to $P_{4}$ and $\mu(G)=\mu\left(H_{1}\right)+\mu\left(G-H_{1}\right)$. Therefore, by Lemma 4.6, $\mathcal{E}(\Phi)>2 \mu(G)$, a contradiction. Thus every vertex in $X^{\prime}$ is adjacent with $v$.

Suppose $u$ is not adjacent to some of the vertices of $Y^{\prime}$. Let $y_{1} \in Y^{\prime}$ such that $y_{1} \nsim u$. Let $x_{1} \in X^{\prime}$. Then the induced subgraph formed by the vertices $\left\{y_{1}, x_{1}, u, v\right\}$ is isomorphic to $P_{4}$. Then by an argument similar to above, we can show that $\mathcal{E}(\Phi)>2 \mu(G)$. Again we get a contradiction. Therefore, $X^{\prime} \subset N(v)$ and $Y^{\prime} \subset N(u)$. Consider $x_{3} \in X^{\prime}$ and $y_{3} \in Y^{\prime}$. Take the induced subgraph $H_{2}$ formed by the vertices $\left\{u, v, u^{\prime}, v^{\prime}, x_{3}, y_{3}\right\}$ which is given in the Figure 2 and of the form shown in Figure 1. Then $\mu(G)=\mu\left(H_{2}\right)+\mu\left(G-H_{2}\right)$. Also by the Lemma 4.7, $\mathcal{E}\left(\left(H_{2}, \varphi\right)\right)>2 \mu\left(H_{2}\right)$. Therefore, by the Lemma 4.6, $\mathcal{E}(\Phi)>2 \mu(G)$. Which is a contradiction. Thus $N(u)=Y$. Therefore, $G=K_{\frac{n}{2}, \frac{n}{2}}$.

Claim 2: $\varphi=1$.

Since $G=K_{\frac{n}{2}, \frac{n}{2}}$, so $\mu(G)=\frac{n}{2}$. Then $\Phi=\left(K_{\frac{n}{2}, \frac{n}{2}}, \varphi\right)$ with $\mathcal{E}(\Phi)=n=\mathcal{E}\left(K_{\frac{n}{2}, \frac{n}{2}}\right)$. Therefore, by the Theorem $3.3, \Phi \sim\left(K_{\left.\frac{n}{2}, \frac{n}{2}, 1\right)}\right.$.

A $k$-walk (or simply walk) in an undirected graph $G$ with vertex set $V(G)=\left\{v_{1}, v_{2}, \ldots, v_{s}\right\}$ is an alternative sequence of vertices and edges. We simply denote $v_{i_{1}}-v_{i_{2}}-\cdots-v_{i_{r}}$ as a $r$-walk from the vertex $v_{i_{1}}$ to $v_{i_{r}}$, where the vertices and edges in this walk may or may not be distinct. We call a walk $v_{i_{1}}-v_{i_{2}}-\cdots-v_{i_{r}}$, a path if all the edges in this walk are distinct. If there is a path in between the vertices $v_{x}$ and $v_{y}$, then we call $v_{x}$ and $v_{y}$ is connected and denoted by $v_{x} \leftrightarrow v_{y}$. 
Let $G_{1}$ and $G_{2}$ be two undirected graph with $V\left(G_{1}\right)=\left\{v_{1}, v_{2}, \ldots, v_{s}\right\}$ and $V\left(G_{2}\right)=$ $\left\{u_{1}, u_{2}, \ldots, u_{t}\right\}$. To avoid the confusion, in definition of Kronecker product, we use the following notation. If $v_{i} \sim v_{j}$, then the undirected edge in between them is denoted by $v_{i} v_{j}$ and the oriented edge from the vertex $v_{i}$ to $v_{j}$ is denoted by $\left(\overrightarrow{v_{i} v_{j}}\right)$. Let $\Phi=\left(G_{1}, \varphi\right)$ be any $\mathbb{T}$-gain graph. A $\mathbb{T}$-gain Kronecker product of $\Phi$ and a simple graph $G_{2}$ is defined as a $\mathbb{T}$-gain graph, $\Phi \otimes G_{2}=\left(G_{1} \otimes G_{2}, \psi\right)$ on an underlying graph $G_{1} \otimes G_{2}$ with vertex set $V\left(G_{1} \otimes G_{2}\right)=\left\{\left(v_{p}, u_{q}\right): p=1,2, \ldots, s\right.$, and $\left.q=1,2, \ldots, t\right\}$ and edge set $E\left(G_{1} \otimes G_{2}\right)=$ $\left\{\left(v_{p}, u_{q}\right)\left(v_{a}, u_{b}\right): v_{p} \sim v_{a}\right.$ and $\left.u_{q} \sim u_{b}\right\}$ such that $\psi\left(\overrightarrow{\left(v_{p}, u_{q}\right)\left(v_{a}, u_{b}\right)}\right)=\varphi\left(\overrightarrow{v_{p} v_{a}}\right)$. The $\mathbb{T}$-gain graph $\Phi \otimes K_{2}$ is called $\mathbb{T}$-gain bipartite double, where $K_{2}$ is a complete graph of 2 vertices. We illustrate the following example of a $\mathbb{T}$-gain bipartite double.

Example 4.1. Let $G$ be a triangle with vertex set $V(G)=\left\{v_{1}, v_{2}, v_{3}\right\}$ and $V\left(K_{2}\right)=\{x, y\}$. Let $\Phi=(G, \varphi)$ be a $\mathbb{T}$-gain graph. Then $\Phi \otimes K_{2}$ is a $\mathbb{T}$-gain bipartite double. See Figure 3 .
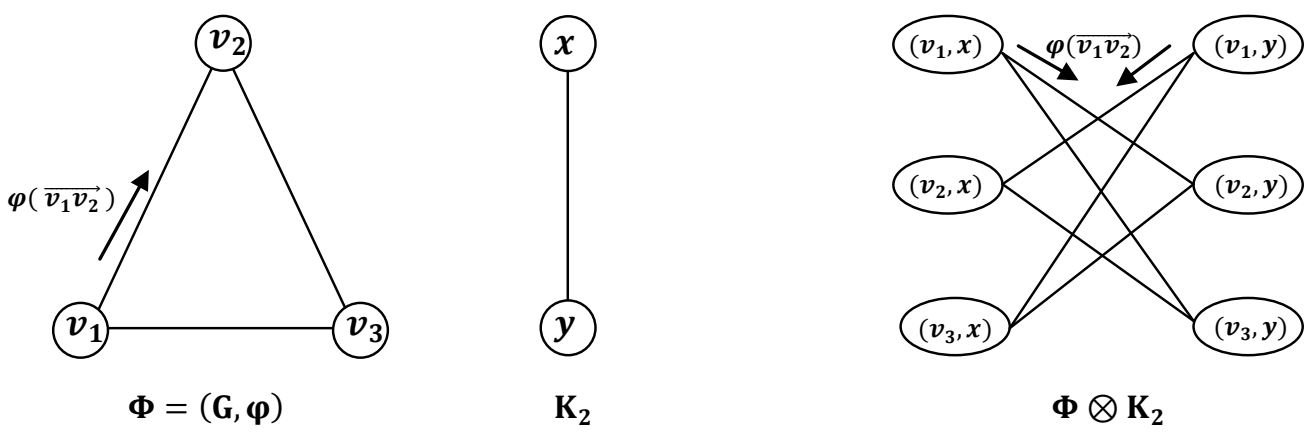

Figure 3: $\mathbb{T}$-gain bipartite double of $\Phi$ and $K_{2}$

For any two matrices $P=\left(p_{i j}\right)_{r_{1} \times r_{2}}$ and $Q=\left(q_{s t}\right)_{s_{1} \times s_{2}}$, the Kronecker product of the matrices $P$ and $Q$ are defined as $P \otimes Q=\left(p_{i j} Q\right)_{r_{1} s_{1} \times r_{2} s_{2}}$. Now, it is easy to see that $A\left(\Phi \otimes G_{2}\right)=A(\Phi) \otimes A\left(G_{2}\right)$.

The following lemma is an extension of Lemma 2.6 for the $\mathbb{T}$-gain graphs.

Lemma 4.8. Let $\Phi \otimes G$ be a $\mathbb{T}$-gain Kronecker product of a $\mathbb{T}$-gain graph $\Phi=\left(G_{1}, \varphi\right)$ and an undirected graph $G$. If $\operatorname{spec}(\Phi)=\left\{\lambda_{1}, \lambda_{2}, \ldots, \lambda_{s}\right\}$ and $\operatorname{spec}(G)=\left\{\gamma_{1}, \gamma_{2}, \ldots, \gamma_{t}\right\}$. Then $\operatorname{spec}(\Phi \otimes G)=\left\{\lambda_{i} \gamma_{j}: i=1,2, \ldots, s, j=1,2, \ldots, t\right\}$.

The following lemma is an extension of Lemma 2.8 for the $\mathbb{T}$-gain graphs. 
Lemma 4.9. If $\Phi=(G, \varphi)$ be any connected $\mathbb{T}$-gain graph on a non bipartite graph $G$, then $\mathcal{E}(\Phi)>2 \mu(G)$.

Proof. Let $\Phi=(G, \varphi)$ be a connected $\mathbb{T}$-gain graph on a non bipartite graph $G$ with vertex set $V(G)=\left\{v_{1}, v_{2}, \ldots, v_{n}\right\}$ and $m$ edges. If possible let $\mathcal{E}(\Phi)=2 \mu(G)$. Then, by Lemma 4.4. $G$ has perfect matching, say $M$. Let $v_{i} v_{j}$ denote the edge between the vertices $v_{i}$ and $v_{j}$, if it exists. Let $M=\left\{v_{1} v_{2}, v_{3} v_{4}, \ldots, v_{n-1} v_{n}\right\}$ be a perfect matching in $G$. Then $\mu(G)=|M|=\frac{n}{2}$. Therefore, $\mathcal{E}(\Phi)=2 \mu(G)=n$. Let $V\left(K_{2}\right)=\{x, y\}$. Now we consider a $\mathbb{T}$-gain Kronecker product $\Phi \otimes K_{2}$. Then $\left|V\left(\Phi \otimes K_{2}\right)\right|=2 n$ and $\left|E\left(\Phi \otimes K_{2}\right)\right|=2 m$. It is easy to see that $\Phi \otimes K_{2}$ is a bipartite graph with a perfect matching $\left\{\left(v_{1}, x\right)\left(v_{2}, y\right),\left(v_{1}, y\right)\left(v_{2}, x\right), \ldots,\left(v_{n-1}, x\right)\left(v_{n}, y\right),\left(v_{n-1}, y\right)\left(v_{n}, x\right)\right\}$. Then $\mu\left(\Phi \otimes K_{2}\right)=n$. Now, by Lemma $4.8, \mathcal{E}\left(\Phi \otimes K_{2}\right)=2 \mathcal{E}(\Phi)=2 n=2 \mu\left(\Phi \otimes K_{2}\right)$. That is, $\mathcal{E}\left(\Phi \otimes K_{2}\right)=2 \mu\left(\Phi \otimes K_{2}\right)$. Claim: $\Phi \otimes K_{2}$ is connected.

The vertex set of $\Phi \otimes K_{2}$ is $V\left(\Phi \otimes K_{2}\right)=\left\{\left(v_{1}, x\right),\left(v_{2}, x\right), \ldots,\left(v_{n}, x\right),\left(v_{1}, y\right),\left(v_{2}, y\right), \ldots,\left(v_{n}, y\right)\right\}$. Since $G$ is connected, so for any pair of vertices $v_{i}$ and $v_{j}$, there is a path in between them, $v_{i}=v_{i_{0}}-v_{i_{1}}-\cdots-v_{i_{t}}=v_{j}$, (say). Now $v_{i}$ and $v_{j}$ is corresponds with the four vertices, $S=\left\{\left(v_{i}, x\right),\left(v_{i}, y\right),\left(v_{j}, x\right),\left(v_{j}, y\right)\right\}$ in $V\left(\Phi \otimes K_{2}\right)$. We show that any pair of two vertices in that four vertices set is connected. If $t$ is even, then we have two paths in $\left(\Phi \otimes K_{2}\right),\left(v_{i}, x\right)=$ $\left(v_{i_{0}}, x\right)-\left(v_{i_{1}}, y\right)-\cdots-\left(v_{i_{t}}, x\right)=\left(v_{j}, x\right)$ and $\left(v_{i}, y\right)=\left(v_{i_{0}}, y\right)-\left(v_{i_{1}}, x\right)-\cdots-\left(v_{i_{t}}, y\right)=\left(v_{j}, y\right)$. Thus $\left(v_{i}, x\right) \leftrightarrow\left(v_{j}, x\right)$ and $\left(v_{i}, y\right) \leftrightarrow\left(v_{j}, y\right)$. If $t$ is odd then similarly, $\left(v_{i}, x\right) \leftrightarrow\left(v_{j}, y\right)$ and $\left(v_{i}, y\right) \leftrightarrow\left(v_{j}, x\right)$. Therefore, it is enough to show that $\left(v_{i}, x\right) \leftrightarrow\left(v_{i}, y\right)$. Since $G$ is connected non bipartite graph, so we can always find a walk from $v_{i}$ to $v_{i}$ of odd length (walk travels an odd cycle). Then similar to above, $\left(v_{i}, x\right) \leftrightarrow\left(v_{i}, y\right)$. Therefore, $\left(v_{i}, x\right)$ is connected with other three vertices of $S$. Since $v_{i}$ and $v_{j}$ are arbitrary pair of vertices of $G$, so any two vertices of $\Phi \otimes K_{2}$ are connected. Thus $\Phi \otimes K_{2}$ is connected.

Since $\Phi \otimes K_{2}$ is a connected bipartite $\mathbb{T}$-gain graph of $2 n$ vertices with $\mathcal{E}\left(\Phi \otimes K_{2}\right)=$ $2 \mu\left(\Phi \otimes K_{2}\right)$, so by Lemma $4.8, \Phi \otimes K_{2} \sim\left(K_{n, n}, 1\right)$. Thus $2 m=\left|K_{n, n}\right|=n^{2}$. That is, $|E(G)|=m=\frac{n^{2}}{2}>\frac{n(n-1)}{2}=\left|E\left(K_{n}\right)\right|$. Which is a contradiction. Hence the result.

Lemma 4.10. Let $\Phi=(G, \varphi)$ be any connected $\mathbb{T}$-gain graph on $n$ vertices with the matching number $\mu(G)$. If $\mathcal{E}(\Phi)=2 \mu(G)$, then $\Phi \sim\left(K_{\frac{n}{2}, \frac{n}{2}}, 1\right)$.

Proof. Since $\mathcal{E}(\Phi)=2 \mu(G)$, so by the Lemma 4.9, $G$ must be bipartite. Therefore, $\Phi=$ $(G, \varphi)$ is a connected bipartite $\mathbb{T}$-gain graph. Now, applying the Theorem 4.6 we have $\Phi \sim\left(K_{\frac{n}{2}, \frac{n}{2}}, 1\right)$. 
Let $A_{1}, A_{2}, \ldots, A_{t}$ be $t$ square complex matrices. Then we denote $A_{1} \oplus A_{2} \oplus \cdots \oplus$ $A_{t}$ as a block diagonal matrix with diagonal blocks are $A_{1}, A_{2}, \ldots, A_{t}$. That is, $\bigoplus_{j=1}^{t} A_{j}=$ $\operatorname{diag}\left(A_{1}, A_{2}, \ldots, A_{t}\right)$.

In the next theorem, we characterize the class of $\mathbb{T}$ - gain graphs for which equality holds in Theorem 4.5.

Theorem 4.7. Let $\Phi=(G, \varphi)$ be any $\mathbb{T}$-gain graph with matching number $\mu(G)$. Then $\mathcal{E}(\Phi)=2 \mu(G)$ if and only if each component of $\Phi$ is a balanced complete bipartite $\mathbb{T}$-gain graph with a perfect matching together with some isolated vertices.

Proof. Let $G_{1}, G_{2}, \ldots, G_{p}, G_{p+1}, \ldots, G_{p+r}$ be the connected components of $G$. Without loss of generality, let us assume that the last $r$ components are the only isolated vertices. Then $\mu(G)=\mu\left(G_{1}\right)+\cdots+\mu\left(G_{p}\right)$. It is clear that $A(\Phi)=\bigoplus_{j=1}^{p+r} A\left(\left(G_{j}, \varphi\right)\right)$, So $\mathcal{E}(\Phi)=\sum_{j=1}^{p} \mathcal{E}\left(\left(G_{j}, \varphi\right)\right)$. Therefore, by the Theorem 4.5 , we have,

$$
2 \mu(G)=\mathcal{E}(\Phi)=\sum_{j=1}^{p} \mathcal{E}\left(\left(G_{j}, \varphi\right)\right) \geq 2 \sum_{j=1}^{p} \mu\left(G_{j}\right)=2 \mu(G)
$$

Thus $\mathcal{E}\left(\left(G_{j}, \varphi\right)\right)=2 \mu\left(G_{j}\right)$, for each $j=1,2, \ldots, s$. Now, using the Lemma 4.10, we can derive the result.

As an application of the above theorem, we can establish a relationship among the energy of $\mathbb{T}$-gain graph, the vertex cover number and the number of odd cycles. This result generalizes one of the main results of [16]. Let $\Phi=(G, \varphi)$ be a $\mathbb{T}$-gain graph with vertex set $V(G)$. Let $u \in V(G)$. Then $(\Phi-u)$ denotes an induced subgraph of $\Phi$ with vertex set $V(G) \backslash\{u\}$.

Theorem 4.8. Let $\Phi=(G, \varphi)$ be any $\mathbb{T}$-gain graph on $G$ with $c(G)$ number of odd cycles and vertex cover number $\tau(G)$. Then

$$
\mathcal{E}(\Phi) \geq 2 \tau(G)-2 c(G)
$$

Equality occurs if and only if each component of $\Phi$ is a balanced complete bipartite $\mathbb{T}$-gain graph with a perfect matching together with some isolated vertices.

Proof. Let $\Phi=(G, \varphi)$ be any $\mathbb{T}$-gain graph with $c(G)$ number of odd cycles. Let us prove the bound using induction on the number of odd cycles $c(G)$. If $c(G)=0$, then $G$ is bipartite. Therefore $\mu(G)=\tau(G)$. Now, by Theorem 4.5, we have $\mathcal{E}(\Phi) \geq 2 \mu(G)=2 \tau(G)-2 c(G)$. 
Assume that the statement is true for any $\mathbb{T}$-gain graph with the number of odd cycles is at most $(c(G)-1)$. Consider $\Phi$ with $c(G) \geq 1$ number of odd cycles. Let $u$ be a vertex in an odd cycle of $G$. Then the number of odd cycles, say $c^{\prime}$, of $\Phi-u$ is at most $(c(G)-1)$. Thus, by induction hypothesis, $\mathcal{E}(\Phi-u) \geq 2 \tau(G-u)-2 c^{\prime}$. Since $u$ is an isolated vertex, so, by Lemma $4.3, \mathcal{E}(\Phi)>\mathcal{E}(\Phi-u)$.

It is easy to see that $\tau(G-u) \geq \tau(G)-1$. Therefore, $\mathcal{E}(\Phi)>\mathcal{E}(\Phi-u) \geq 2 \tau(G)-2 c(G)$.

Now, let $\mathcal{E}(\Phi)=2 \tau(G)-2 c(G)$. If $c(G) \geq 1$, then, by the above observation, $\mathcal{E}(\Phi)>$ $2 \tau(G)-2 c(G)$. which is a contradiction. That is $c(G)=0$. Therefore, $G$ is bipartite and $\mu(G)=\tau(G)$. Thus $\mathcal{E}(\Phi)=2 \mu(G)$. Now, by Theorem 4.6, $\Phi$ is the disjoint union of some balanced complete bipartite $\mathbb{T}$-gain graphs with a perfect matching together with some isolated vertices.

\section{Upper bound of energy of $\mathbb{T}$-gain graph in terms of vertex cover number and largest vertex degree}

In this section, our main objective is to obtain an upper bound for the energy of a $\mathbb{T}$-gain graph in terms of the vertex cover number and the largest vertex degree. This result is the counter part of the corresponding known result about undirected graph [Theorem 1.3] and mixed graph [Theorem 1.4]. Furthermore, we characterize all $\mathbb{T}$-gain graphs for which the upper bound is attained. This characterization completely solve one of the open problem [16].

Theorem 5.1. Let $\Phi=(G, \varphi)$ be any $\mathbb{T}$-gain graph with the vertex cover number $\tau(G)$, and maximum vertex degree $\Delta(G)$. Then,

$$
\mathcal{E}(\Phi) \leq 2 \tau(G) \sqrt{\Delta(G)}
$$

Proof. Let $\Phi=(G, \varphi)$ be any $\mathbb{T}$-gain graph with vertex cover number $\tau(G)$. We prove the result by induction on $\tau(G)$. If $\tau(G)=1$, then $G$ must be $K_{1, r}$, for some $r$ together with some isolated vertices. Therefore, $\Phi$ is balanced. Now $\mathcal{E}(\Phi)=\mathcal{E}\left(K_{1, r}\right)=2 \sqrt{r}=2 \tau(G) \sqrt{\Delta\left(K_{1, r}\right)}$.

Let us assume that for any $\mathbb{T}$-gain graph $\Psi=\left(G_{1}, \psi\right)$ with $\tau\left(G_{1}\right)<\tau(G)$, we have $\mathcal{E}(\Psi) \leq 2 \tau\left(G_{1}\right) \sqrt{\Delta\left(G_{1}\right)}$. Let $U$ be a minimum vertex cover of $G$. Then $|U|=\tau(G) \geq 2$. Let $x \in U$. Let $S$ be an induced subgraph of $G$ which is formed by removing the vertex $x$, and the edges incident with $x$ from $G$. That is $S=G-x$. Then $\tau(S)=\tau(G)-1$. Therefore, by

the induction hypothesis, $\mathcal{E}(\Phi-x)=\mathcal{E}((S, \varphi)) \leq 2 \tau(S) \sqrt{\Delta(S)}$. After a suitable relabeling 
of vertices, we can express $A(\Phi)$ as

$$
A(\Phi)=\left[\begin{array}{ccc}
0 & \mathbf{v}^{*} & \mathbf{0} \\
\mathbf{v} & A_{1} & Y^{*} \\
\mathbf{0} & Y & A_{2}
\end{array}\right]=\left[\begin{array}{ccc}
0 & \mathbf{v}^{*} & \mathbf{0} \\
\mathbf{v} & \mathbf{0} & \mathbf{0} \\
\mathbf{0} & \mathbf{0} & \mathbf{0}
\end{array}\right]+\left[\begin{array}{ccc}
0 & \mathbf{0} & \mathbf{0} \\
\mathbf{0} & A_{1} & Y^{*} \\
\mathbf{0} & Y & A_{2}
\end{array}\right]
$$

Here the first column and the first row are associated with the vertex $x$. Let the degree of $x$ be $d$. Then $\left[\begin{array}{cc}0 & \mathbf{v}^{*} \\ \mathbf{v} & \mathbf{0}\end{array}\right]$ and $\left[\begin{array}{cc}A_{1} & Y^{*} \\ Y & A_{2}\end{array}\right]$ are the adjacency matrices of the $\mathbb{T}$-gain subgraphs $\left(K_{1, d}, \varphi\right)$ and $(S, \varphi)$, respectively. By Theorem 2.11, we have

$$
\mathcal{E}(\Phi) \leq \mathcal{E}\left(K_{1, d}\right)+\mathcal{E}((S, \varphi)) \leq 2 \sqrt{d}+2 \tau(S) \sqrt{\Delta(S)} \leq 2 \tau(G) \sqrt{\Delta(G)} .
$$

Theorem 5.2. Let $\Phi=(G, \varphi)$ be any $\mathbb{T}$-gain graph on $G$ with vertex cover number $\tau(G)$ and maximum vertex degree $\Delta(G)$. Then

$$
\mathcal{E}(\Phi)=2 \tau(G) \sqrt{\Delta(G)}
$$

if and only if $\Phi$ is the disjoint union of $\tau(G)$ copies of balanced $\mathbb{T}$-gain graph $\left(K_{1, \Delta(G)}, 1\right)$ together with some isolated vertices.

Proof. First let us show that all the vertices of $U$ have the same vertex degree, $\Delta(G)$. Let $x \in U$ be any vertex in $U$ (as in Theorem 5.1). Since $\mathcal{E}(\Phi)=2 \tau(G) \sqrt{\Delta(G)}$, so all the inequalities of 15 become equations. So $\mathcal{E}((S, \varphi))=2 \tau(S) \sqrt{\Delta(S)}$, and $d=\Delta(S)=\Delta(G)$. As $x$ is arbitrary, so all the vertices of $U$ are of degree $\Delta(G)$.

Now we claim that the underlying graph $G$ is bipartite. Let $W=V(G) \backslash U$. It is clear that $U \backslash\{x\}$ is a minimum vertex cover of the induced subgraph $S$. Also, we have $\mathcal{E}((S, \varphi))=$ $2 \tau(S) \sqrt{\Delta(S)}$. Now, applying the argument

to $S$. Therefore, all the vertices of $U \backslash\{x\}$ in $S$ is of degree $\Delta(S)$. Also we know that $\Delta(S)=\Delta(G)$. Since $d=\Delta(G)$, so there is no edge between the vertex $x$ and the vertices of $U \backslash\{x\}$. As $x$ is arbitrary, so we get no two vertices of $U$ are adjacent. Now $U$ is a minimum vertex cover of $G$, so no two vertices of $W$ are adjacent. Hence $G$ is a bipartite graph with vertex partition sets $U$ and $W$.

Let $G_{1}, G_{2}, \ldots, G_{p}$ be the only nontrivial components of $G$ (That is components contain at least one edge). Then,

$$
2 \tau(G) \sqrt{\Delta(G)}=\mathcal{E}(\Phi)=\sum_{j=1}^{p} \mathcal{E}\left(\left(G_{j}, \varphi\right)\right) \leq \sum_{j=1}^{p} 2 \tau\left(G_{j}\right) \sqrt{\Delta\left(G_{j}\right)} \leq 2 \tau(G) \sqrt{\Delta(G)}
$$


From the above expression, we get $\mathcal{E}\left(\left(G_{j}, \varphi\right)\right)=2 \tau\left(G_{j}\right) \sqrt{\Delta\left(G_{j}\right)}$ and $\Delta\left(G_{j}\right)=\Delta(G)$, for $j=1,2, \ldots, p$.

Now let us show that the rank of each component $\left(G_{j}, \varphi\right)$ is 2 . Let $r_{j}$ be the rank of $\left(G_{j}, \varphi\right)$. Since $\left(G_{j}, \varphi\right)$ is bipartite, so its spectrum is symmetric with respect to origin. Thus $r_{j}$ is an even number and $r_{j} \geq 2$. Let $\lambda_{1} \geq \lambda_{2} \geq \cdots \geq \lambda_{r_{j}}$ be the nonzero eigenvalues of $\left(G_{j}, \varphi\right)$. Suppose that $r_{j}>2$. Then $\lambda_{1}>\lambda_{2}>0$. Therefore, by the Cauchy-Schwartz inequality

$$
\mathcal{E}\left(\left(G_{j}, \varphi\right)\right)=\sum_{t=1}^{j}\left|\lambda_{t}\right|<\sqrt{r_{j}} \sqrt{\sum_{t=1}^{j} \lambda_{t}^{2}}=\sqrt{2\left|E\left(G_{j}\right)\right| r_{j}} .
$$

For any $\mathbb{T}$-gain graph $\Psi=(B, \psi)$ on a bipartite graph $B$, we know that $|E(B)| \leq \tau(B) \Delta(B)$. By Lemma 2.3, we have $\operatorname{rank}(\Psi) \leq 2 \mu(B)=2 \tau(B)$. Hence $\mathcal{E}\left(\left(G_{j}, \varphi\right)\right)<2 \tau\left(G_{j}\right) \sqrt{\Delta\left(G_{j}\right)}$, a contradiction (as for each component, $\mathcal{E}\left(\left(G_{j}, \varphi\right)\right)=2 \tau\left(G_{j}\right) \sqrt{\Delta\left(G_{j}\right)}$ ). Hence the rank of $\left(G_{j}, \varphi\right)$ is 2 for $j=1,2, \cdots, p$.

Since each nontrivial component $\left(G_{j}, \varphi\right)$ is bipartite and of rank 2. Now $\left(G_{j}, \varphi\right)$ is of rank 2 if and only if it has exactly one positive eigenvalue. Therefore, by Lemma 4.2. $\left(G_{j}, \varphi\right) \sim\left(K_{a, b}, 1\right)$. Without loss of generality, consider $a \leq b$. Then $\tau\left(G_{j}\right)=a$ and $\Delta\left(G_{j}\right)=b$. Now $\mathcal{E}\left(\left(G_{j}, \varphi\right)\right)=2 \tau\left(G_{j}\right) \sqrt{\Delta\left(G_{j}\right)}=2 \tau\left(G_{j}\right) \sqrt{\Delta\left(G_{j}\right)}=2 a \sqrt{b}$. On the other hand $\mathcal{E}\left(\left(G_{j}, \varphi\right)\right)=\mathcal{E}\left(\left(K_{a, b}, 1\right)\right)=2 \sqrt{a b}$. Thus $2 a \sqrt{b}=2 \sqrt{a b}$. Thus $a=1$, and hence $b=\Delta\left(G_{j}\right)=\Delta(G)$. Therefore, for each $j=1,2, \ldots, p,\left(G_{j}, \varphi\right) \sim\left(K_{1, \Delta(G)}, 1\right)$.

\section{Acknowledgments}

Aniruddha Samanta thanks University Grants Commission(UGC) for the financial support in the form of the Senior Research Fellowship (Ref.No: 19/06/2016(i)EU-V; Roll No. 423206). M. Rajesh Kannan would like to thank the SERB, Department of Science and Technology, India, for financial support through the projects MATRICS (MTR/2018/000986) and Early Career Research Award (ECR/2017/000643).

\section{References}

[1] Octavio Arizmendi, Jorge Fernandez Hidalgo, and Oliver Juarez-Romero, Energy of a vertex, Linear Algebra Appl. 557 (2018), 464-495. MR 3848283 
[2] Che-Man Cheng, Roger A. Horn, and Chi-Kwong Li, Inequalities and equalities for the cartesian decomposition of complex matrices, Linear Algebra and its Applications 341 (2002), no. 1, 219 - 237, Special issue dedicated to Professor T. Ando.

[3] Jane Day and Wasin So, Graph energy change due to edge deletion, Linear Algebra and its Applications 428 (2008), no. 8, 2070 - 2078.

[4] Krystal Guo and Bojan Mohar, Hermitian adjacency matrix of digraphs and mixed graphs, J. Graph Theory 85 (2017), no. 1, 217-248. MR 3634484

[5] I. Gutman, S.Z. Firoozabadi, J.A. De La Pea, and J. Rada, On the energy of regular graphs, Match 57 (2007), no. 2, 435-442, cited By 50.

[6] Shengjie He, Rong-Xia Hao, and Fengming Dong, The rank of a complex unit gain graph in terms of the matching number, Linear Algebra and its Applications 589 (2020), 158 -185 .

[7] Roger A. Horn and Charles R. Johnson, Matrix analysis, second ed., Cambridge University Press, Cambridge, 2013. MR 2978290

[8] Yuxuan Li, Lower bound of the energy of a complex unit gain graph in terms of the matching number of its underlying graph, arXiv preprint arXiv:2005.01998 [math.CO] (2020).

[9] Jianxi Liu and Xueliang Li, Hermitian-adjacency matrices and Hermitian energies of mixed graphs, Linear Algebra Appl. 466 (2015), 182-207. MR 3278246

[10] Ranjit Mehatari, M Rajesh Kannan, and Aniruddha Samanta, On the adjacency matrix of complex unit gain graph, arXiv preprint arXiv:1812.03747 [math.CO] (2018).

[11] Nathan Reff, Spectral properties of complex unit gain graphs, Linear Algebra Appl. 436 (2012), no. 9, 3165-3176. MR 2900705

[12] _ Oriented gain graphs, line graphs and eigenvalues, Linear Algebra Appl. 506 (2016), 316-328. MR 3530682

[13] Aniruddha Samanta and M. Rajesh Kannan, On the spectrum of complex unit gain graph, arXiv preprint arXiv:1908.10668 (2019). 
[14] Fenglei Tian and Dein Wong, Relation between the skew energy of an oriented graph and its matching number, Discrete Applied Mathematics 222 (2017), 179 - 184.

[15] Long Wang and Xiaobin Ma, Bounds of graph energy in terms of vertex cover number, Linear Algebra Appl. 517 (2017), 207-216. MR 3592020

[16] W. Wei and S. Li, Relation between the hermitian energy of a mixed graph and the matching number of its underlying graph, Linear and Multilinear Algebra (2018), cited By 1; Article in Press.

[17] Dein Wong, Xinlei Wang, and Rui Chu, Lower bounds of graph energy in terms of matching number, Linear Algebra and its Applications 549 (2018), 276 - 286.

[18] Thomas Zaslavsky, Signed graphs, Discrete Appl. Math. 4 (1982), no. 1, 47-74. MR 676405 